\title{
NOVA-LIKE CATACLYSMIC VARIABLES IN THE INFRARED
}

\author{
D. W. Hoard ${ }^{1,2,15}$, Knox S. Long ${ }^{3}$, Steve B. Howell ${ }^{4}$, Stefanie Wachter ${ }^{2}$, Carolyn S. Brinkworth ${ }^{5,6}$, \\ Christian Knigge $^{7}$, J. E. Drew ${ }^{8}$, Paula Szkody ${ }^{9}$, S. Kafka ${ }^{10}$, Kunegunda Belle $^{11}$, David R. Ciardi ${ }^{6}$, \\ Cynthia S. Froning ${ }^{12}$, Gerard T. van Belle ${ }^{13}$, and M. L. Pretorius ${ }^{14}$ \\ ${ }^{1}$ Eureka Scientific, Inc., Oakland, CA, USA \\ ${ }^{2}$ Max Planck Institut für Astronomie, D-69117 Heidelberg, Germany; hoard@mpia.de \\ ${ }^{3}$ Space Telescope Science Institute, Baltimore, MD, USA \\ ${ }^{4}$ NASA Ames Research Center, Moffett Field, CA, USA \\ ${ }^{5}$ Spitzer Science Center, California Institute of Technology, Pasadena, CA, USA \\ ${ }^{6}$ NASA Exoplanet Science Institute, California Institute of Technology, Pasadena, CA, USA \\ ${ }^{7}$ Physics and Astronomy, University of Southampton, Southampton, UK \\ ${ }^{8}$ Centre for Astrophysics Research, Science and Technology Research Institute, University of Hertfordshire, Hatfield, UK \\ ${ }^{9}$ Department of Astronomy, University of Washington, Seattle, WA, USA \\ ${ }^{10}$ Carnegie Institution of Washington, Department of Terrestrial Magnetism, Washington, DC, USA \\ ${ }^{11}$ Los Alamos National Laboratory, Los Alamos, NM, USA \\ ${ }^{12}$ Center for Astrophysics and Space Astronomy, Department of Astrophysical and Planetary Sciences, \\ University of Colorado, Boulder, CO, USA \\ ${ }^{13}$ Lowell Observatory, Flagstaff, AZ, USA \\ ${ }^{14}$ Department of Physics, University of Oxford, Oxford, UK \\ Received 2013 December 13; accepted 2014 March 20; published 2014 April 16
}

\begin{abstract}
Nova-like (NL) cataclysmic variables have persistently high mass transfer rates and prominent steady state accretion disks. We present an analysis of infrared observations of 12 NLs obtained from the Two Micron All Sky Survey, the Spitzer Space Telescope, and the Wide-field Infrared Survey Explorer All Sky Survey. The presence of an infrared excess at $\lambda \gtrsim 3-5 \mu \mathrm{m}$ over the expectation of a theoretical steady state accretion disk is ubiquitous in our sample. The strength of the infrared excess is not correlated with orbital period, but shows a statistically significant correlation (but shallow trend) with system inclination that might be partially (but not completely) linked to the increasing view of the cooler outer accretion disk and disk rim at higher inclinations. We discuss the possible origin of the infrared excess in terms of emission from bremsstrahlung or circumbinary dust, with either mechanism facilitated by the mass outflows (e.g., disk wind/corona, accretion stream overflow, and so on) present in NLs. Our comparison of the relative advantages and disadvantages of either mechanism for explaining the observations suggests that the situation is rather ambiguous, largely circumstantial, and in need of stricter observational constraints.
\end{abstract}

Key words: accretion, accretion disks - circumstellar matter - infrared: stars - novae, cataclysmic variables stars: individual (TT Ari, WX Ari, QU Car, V592 Cas, V442 Oph, V347 Pup, V3885 Sgr, VY Scl, RW Sex, RW Tri, UX UMa, IX Vel)

\section{INTRODUCTION}

Cataclysmic variables (CVs) are interacting binary stars containing an accreting white dwarf (WD) primary and a masslosing, late-type secondary star that fills its Roche lobe. CVs are a common pathway for binary star evolution that includes classical novae and possibly leads to the standard candlesType Ia supernovae-that play a crucial role in modern cosmology (e.g., see Bours et al. 2013). In most CVs, accretion of matter from the secondary star onto the (non-magnetic) WD is mediated by a disk that extends close to the surface of the WD. CVs arguably represent the most observationally accessible disk-accreting astrophysical systems. They also include the closest examples of an accretion flow around a compact object and, hence, provide a basic laboratory for accretion disk physics that is relevant in fields ranging from star and planet formation to the central engines of quasars and active galactic nuclei. See Warner (2003) for a thorough review of CV types and observational characteristics.

All CVs vary in mean brightness on a number of characteristic timescales and amplitudes, but the overall nature of the variability probably reflects the time-averaged mass transfer rate from

\footnotetext{
${ }^{15}$ Visiting Scientist, MPIA.
}

the secondary star. Systems with mass transfer rates below a critical value $\left(\lesssim 10^{-9} M_{\odot} \mathrm{yr}^{-1}\right.$ for orbital periods $\lesssim 8 \mathrm{hr}$; e.g., Patterson 1984) show quasiperiodic outbursts of 3-5 mag (in visible light) that last days to weeks and recur on timescales of weeks to months to (in extreme cases) years. These CVs are known as dwarf novae (DNe). The outbursts of DNe are due to a thermal instability that converts the geometrically thin disk from a low temperature, mostly unionized state to a high temperature, ionized, optically thick state (Cannizzo et al. 1988; Osaki 1996; Lasota 2001). During a DN outburst, the mass transfer rate in the inner disk rises to $\gtrsim 10^{-9} M_{\odot} \mathrm{yr}^{-1}$ (Hameury et al. 1998).

In contrast, CVs with persistently high mass transfer rates ( $1^{-9} M_{\odot} \mathrm{yr}^{-1}$; Patterson 1984; Ballouz \& Sion 2009) remain in the high temperature state most of the time and have prominent, optically thick accretion disks that do not display outbursts. These systems are known as nova-likes (NLs). Because they are almost always in the high temperature state, NLs provide a valuable opportunity to study a prototypical steady state accretion disk.

Disk-dominated CVs have been studied extensively in X-rays, the ultraviolet (UV), and visible wavelengths, where they exhibit different behavior depending on the sub-type and brightness state of the system. A variety of components contribute to the spectral energy distributions (SEDs) of CVs, including the WD 
onto which matter is being accreted, the accretion disk itself, the boundary layer between the WD and the disk (mainly in the UV and shorter wavelengths), the interaction region where matter from the secondary star is entrained into the disk, and the secondary star itself. When the accretion rate is high, emission from the far-UV to the infrared (IR) is dominated by the accretion disk (e.g., see Hoard et al. 2009 and Section 3.3).

Hoard et al. (2009) used multi-wavelength archival and Spitzer Space Telescope Cycle-2 IR observations of the lowinclination (near face-on) NL V592 Cassiopeiae to construct an SED and system model from the UV to the IR. They showed that there is an excess of flux density in the IR that is not reproduced by the usual complement of CV components (WD, accretion disk, secondary star). They modeled this IR excess with a circumbinary dust disk.

Through the gravitational torques that would be exerted on the central binary, circumbinary dust disks were proposed as an additional route for angular momentum loss driving the secular evolution of CVs (Spruit \& Taam 2001; Taam \& Spruit 2001; Dubus et al. 2002; Taam et al. 2003; Belle et al. 2004; Willems et al. 2005, 2007). However, Hoard et al. (2009) found that the implied mass of dust in V592 Cas $\left(\sim 10^{21} \mathrm{~g}\right)$ was many orders of magnitude too small to be effective in that regard. ${ }^{16}$ The importance of the contribution of a circumbinary dust disk to the SEDs of CVs (or even the presence of dust in CVs at all) is not yet firmly established nor universally accepted. Alternative explanations for an IR excess in CVs have been proposed (e.g., bremsstrahlung; see Harrison et al. 2013 and the discussion herein).

We report here on our Spitzer observations of a sample of 11 additional NLs. These observations were obtained in order to study the general properties of CVs in the mid-IR; NLs were selected as targets to ensure the presence of bright accretion disks and avoid the complications imposed by DN outbursts or strong magnetic fields in other types of CV. At the same time, these NLs offer an opportunity to explore the potential observational consequences of the mass outflows from the inner binary expected in high mass transfer rate systems (e.g., disk corona/wind, stream overflow, and so on). Because these mechanisms for relocating matter out of the WD Roche lobe and into circumbinary space operate strongly in NLs, these $\mathrm{CVs}$ are prime candidates for probing for the presence of dust.

\section{TARGETS AND OBSERVATIONS}

Our primary targets are the nine brightest (in visible/nearIR light) NLs, which were observed in our Spitzer Cycle-5 program (50068). From brightest to faintest in $K s$ band, they are: IX Velorum, V3885 Sagittarii, RW Sextantis, QU Carinae, TT

\footnotetext{
16 Recent discoveries of cyclic eclipse timing variations interpreted as evidence of circumbinary planets around several CVs and pre-CVs (e.g., RR Caeli-Qian et al. 2012a; UZ Fornacis-Potter et al. 2011; DP Leonis-Qian et al. 2010a; Beuermann et al. 2011; NN Serpentis-Brinkworth et al. 2006; Parsons et al. 2014; Marsh et al. 2014; HW Virginis-Lee et al. 2009; NY Virginis_Qian et al. 2012b; QS Virginis_-Parsons et al. 2010; Qian et al. 2010b) have possibly resurrected this scenario, since the added mass of a planet could increase the gravitational torques on the inner binary to levels sufficient to affect secular evolution. However, the veracity of the planet detections is still being debated in the literature (e.g., HU Aquarii; see Schwarz et al. 2009; Qian et al. 2011; Horner et al. 2011; Goździewski et al. 2012; Hinse et al. 2012; Wittenmyer et al. 2012). Certainly the existence of circumbinary planets as a general class of object has now been firmly established by discoveries from Kepler (e.g., Kepler-34 and Kepler-35, Welsh et al. 2012; Kepler-16, Doyle et al. 2011; Kepler-38, Orosz et al. 2012a; Kepler-47, Orosz et al. 2012b), so the possibility that a CV could host a circumbinary planet should not be casually discarded.
}

Arietis, RW Trianguli, UX Ursae Majoris, V347 Puppis, and VY Sculptoris. In addition, we utilized a serendipitous observation of UX UMa from Spitzer program 40204. The bulk of our data for IX Vel was actually obtained as part of our Cycle-2 program (20221), with only a repeat spectroscopic measurement during Cycle-5. We also include two additional NLs from our Cycle2 program, WX Arietis and V442 Ophiuchi. Although fainter than the Cycle-5 program targets, these objects are intermediate inclination members of the SW Sextantis sub-type of CV (as is TT Ari, and probably also RW Tri, UX UMa, and V347 Pup). The SW Sex stars are NLs that share a number of unusual observational characteristics (Honeycutt et al. 1986; Szkody \& Piche 1990; Thorstensen et al. 1991) that are likely linked to the presence of a self-occulting, flared accretion disk with a bright spot at the impact site of the matter stream from the secondary star with the disk edge (Hoard et al. 2003, 2010b; Dhillon et al. 2013). ${ }^{17}$ Although not re-observed as part of our Spitzer Cycle-5 program, V592 Cas from our Cycle-2 program is included here as a baseline for comparison to a CV with known IR excess.

These targets span a wide range of orbital period and inclination (see Table 1). To first order, longer orbital period correlates with higher mass transfer rate from the secondary star (Patterson 1984; Howell et al. 2001), in principle allowing us to probe the effect of differing rates of mass flow through the accretion disk and self-irradiation via accretion-generated luminosity close to the WD. The lower inclination (near face-on) $\mathrm{CV}$ s provide a view of the entire radial profile of the accretion disk, as well as the maximum projected disk surface area. Thus, they potentially offer the most sensitive probes of departures of the disk SED from the steady state prediction.

Table 2 lists the log of Spitzer observations and other archival data for each target. We utilized all of Spitzer's instruments during this observing campaign. Depending on target brightness, this provided photometric data in up to six bands from the Infrared Array Camera (IRAC; IRAC-1-IRAC-4 bands at 3.55, 4.5, 5.7, $7.9 \mu \mathrm{m})$, the Infrared Spectrograph Peak-Up Imaging array (IRSPUI; blue or red band at 15.8 or $22.3 \mu \mathrm{m}$ ), and the Multi-band Imaging Photometer for Spitzer (MIPS; MIPS-24 band at $23.7 \mu \mathrm{m}) .{ }^{18}$ For seven of the targets, we also obtained Infrared Spectrograph (IRS) low resolution spectra, spanning wavelengths from $\approx 5 \mu \mathrm{m}$ to (depending on target brightness) $\approx 8,14$, or $20 \mu \mathrm{m}$.

In addition to the Spitzer data, we utilized IR photometry from the Two-Micron All Sky Survey (2MASS, JHKs bands; Skrutskie et al. 2006) and the Wide-field Infrared Survey Explorer (WISE) All Sky Survey (Wright et al. 2010). WISE provides photometric data in four bands, denoted $W 1, W 2, W 3$, and $W 4$, at wavelengths of $3.35,4.6,11.6$, and $22.1 \mu \mathrm{m}$, respectively. When available, the Spitzer observations generally reach greater sensitivity (hence, improved photometric accuracy) compared to WISE. ${ }^{19}$ The latter are useful to provide confirmation of the

\footnotetext{
17 Also see D. W. Hoard's Big List of SW Sextantis Stars at http://www.dwhoard.com/biglist.

18 See the corresponding Instrument Handbooks for details of each instrument, http://irsa.ipac.caltech.edu/data/SPITZER/docs/irac/iracinstrumenthandbook/, http://irsa.ipac.caltech.edu/data/SPITZER/docs/irs/irsinstrumenthandbook/, and http://irsa.ipac.caltech.edu/data/SPITZER/docs/mips/ mipsinstrumenthandbook.

19 The nominal $5 \sigma$ point source sensitivity limits for the WISE All Sky Survey are $0.08,0.11,1$, and $6 \mathrm{mJy}$ in $W 1, W 2, W 3$, and $W 4$, respectively (Wright et al. 2010); cold Spitzer surpassed these sensitivity limits for IRAC-1 $(\approx W 1)$, IRAC-2 $(\approx W 2)$, and MIPS-24 $(\approx W 4)$ in total on-source times of less than a minute (see the Spitzer Performance Estimation Tool at http://ssc.spitzer.caltech.edu/warmmission/propkit/pet/senspet/index.html).
} 
Table 1

Target Information

\begin{tabular}{lccc}
\hline \hline Target & $\begin{array}{c}\text { Distance } \\
(d ; \mathrm{pc})\end{array}$ & $\begin{array}{c}\text { Orbital Period } \\
\left(P_{\text {orb }} ; \mathrm{d}\right)\end{array}$ & $\begin{array}{c}\text { Inclination } \\
\left(i ;{ }^{\circ}\right)\end{array}$ \\
\hline V592 Cas & $380[1]$ & $0.115063(1)[2]$ & $28_{-11}^{+11}[2]$ \\
IX Vel & $96(9)[3]$ & $0.193929(2)[4]$ & $57(2)[3]$ \\
QU Car & $\sim 610[5]$ & $0.454(14)[6]$ & $40-55[5]$ \\
RW Sex & $150,289[7]$ & $0.24507(20)[8]$ & $34(6)[7]$ \\
TT Ari & $335(50)[9]$ & $0.13755040(17)[10]$ & $29(6)[10]$ \\
RW Tri & $470(130)[14]$ & $0.231883297[11]$ & $75(+5,-8)[13]$ \\
V347 Pup & $250-345[15], 345(34)[13]$ & $0.231936060(6)[14]$ & $84(2)[14]$ \\
UX UMa & $110(30)[17]$ & $0.196671278(2)[16]$ & $70.2(2)[15], 71.0(6)[13], 65-75[16]$ \\
V3885 Sgr & $536-620[19]$ & $0.20716071(22)[18]$ & $65(2)[17]$ \\
VY Scl & $183[21]$ & $0.232(3)[20]$ & $30(10)[20], 41[19]$ \\
V442 Oph & $468[21]$ & $0.12435(7)[22]$ & $70(5)[21,22]$ \\
WX Ari & & $0.13935119(3)[23]$ & $\approx 60[21], \approx 72[23]$ \\
\hline
\end{tabular}

References. Listed in brackets with each parameter value: $1=$ this work; $2=$ Hoard et al. (2009); $3=$ Linnell et al. (2007a); $4=$ Beuermann \& Thomas (1990); $5=$ Linnell et al. (2008b); $6=$ Gilliland \& Phillips (1982); $7=$ Linnell et al. (2010)—the smaller distance is from Bailey's method and yields the favored model, while the larger distance is from the Hipparcos parallax and has a $1 \sigma$ lower limit of $169 \mathrm{pc} ; 8=\mathrm{Hillwig}$ et al. (2004); $9=$ Gänsicke et al. (1999); $10=$ Wu et al. (2002); $11=$ Groot et al. (2004); $12=$ McArthur et al. (1999)—distance from Hubble Space Telescope Fine Guidance Sensor trigonometric parallax; 13 = Noebauer et al. (2010); $14=$ Thoroughgood et al. (2005); $15=$ Linnell et al. (2008a); 16 = Neustroev et al. (2011); 17 = Linnell et al. (2009)—distance is from the Hipparcos parallax; 18 = Ribeiro \& Diaz (2007); 19 = Hamilton \& Sion (2008); 20 = Martínez-Pais et al. (2000); 21 = Ballouz \& Sion (2009); 22 = Hoard et al. (2000); $23=$ Rodríguez-Gil et al. (2000).

Spitzer photometry, a second epoch measurement, and/or coverage at wavelengths not sampled by Spitzer observations.

\subsection{Photometric and Spectroscopic Data Extraction}

In most cases, we utilized aperture photometry performed with IRAF ${ }^{20}$ (version 2.16; method as described in Hoard et al. 2009) to extract the Spitzer photometry from the corrected basic calibrated data IRAC images, the standard BCD IRSPUI images, and the enhanced BCD (EBCD) MIPS images. We generally utilized the smallest calibrated aperture case whenever possible (as described in the corresponding Instrument Handbooks). We also performed aperture and point response function (PRF)fitting photometry with MOPEX/APEX ${ }^{21}$ as a check of the IRAF results for IRAC and IRSPUI whenever the presence of nearby sources might have affected the aperture photometry. We generally preferred MOPEX/APEX aperture photometry for the MIPS observations in order to fully utilize the mosaicking properties of the EBCD images. The final photometry, along with the method used to procure it from each instrument for each target, are listed in Table 3 (and plotted in Figures 1 and 2; see discussion in Section 3.1); pertinent notes are described on a target-by-target basis below. We transformed the cataloged 2MASS and WISE photometry from magnitudes to flux densities using the zeropoint information in Cohen et al. (2003) and Jarrett et al. (2011), respectively.

For the targets with Spitzer IRS observations, we extracted spectra from the post-BCD pipeline images using the standard procedure with the SPICE ${ }^{22}$ software (version 2.3F). Spectra were extracted from each nod individually, then combined using a weighted average. For plotting purposes, the spectroscopic data were block-averaged in wavelength bins of two points.

\footnotetext{
20 IRAF is distributed by the National Optical Astronomy Observatory, which is operated by the Association of Universities for Research in Astronomy (AURA) under cooperative agreement with the National Science Foundation.

21 See http://irsa.ipac.caltech.edu/data/SPITZER/docs/dataanalysistools/ tools/mopex/mopexusersguide/.

22 See http://irsa.ipac.caltech.edu/data/SPITZER/docs/dataanalysistools/ tools/spice/spiceusersguide/.
}

Because CVs are intrinsically variable objects, one must be cautious when combining multiple data sets obtained at (significantly) different times. The NLs studied here, in particular, are subject to stochastic brightness variations with amplitude up to several tenths of a magnitude across a wide range of timescales, as well as transitions to low mass transfer states resulting in sustained drops in overall brightness (see Appendix A.9). Whenever possible, we attempted to mitigate the effects of variability between data sets by consulting available brightness information from published sources or the American Association of Variable Star Observers (AAVSO) Light Curve Generator, ${ }^{23}$ or by comparing overlapping data obtained at similar wavelengths but different times. Unfortunately, there is no ex post facto means of precisely evaluating the relative effect of stochastic brightness variations on approximately orbital timescales in non-simultaneous archival data sets. However, the amplitude of this type of variability is substantially smaller than that of the long-term brightness state changes in NLs and, to some extent, it should "average out" when utilizing multiple data points sampled over intervals up to a few orbits in duration.

When brightness offsets between overlapping data sets existed, we determined a correction to the fainter data to bring it into agreement with the brighter data. This was accomplished by calculating a Rayleigh-Jeans-like (RJL) offset function, in which an additive offset is used to match the shortest wavelength point in the faint data to the corresponding point in the bright data, then scaling this offset by $\lambda^{-2}$ at each longer wavelength point (i.e., the offset function is $\Delta f_{v}(\lambda)=\Delta f_{v, 0} \lambda^{-2}$ ). Physically, this correction implicitly assumes that the variation in total system brightness is primarily caused by a change in the accretion disk, and that the disk dominates the system brightness in the IR - we discuss this in more detail in Section 3.3.2. We have applied offsets conservatively, restricting this procedure only to cases for which there is a clear and significant discrepancy between overlapping data sets and/or an independent confirmation of a change in mean system brightness at one of

\footnotetext{
23 See http://www.aavso.org/lcg.
} 
Table 2

Observation Log

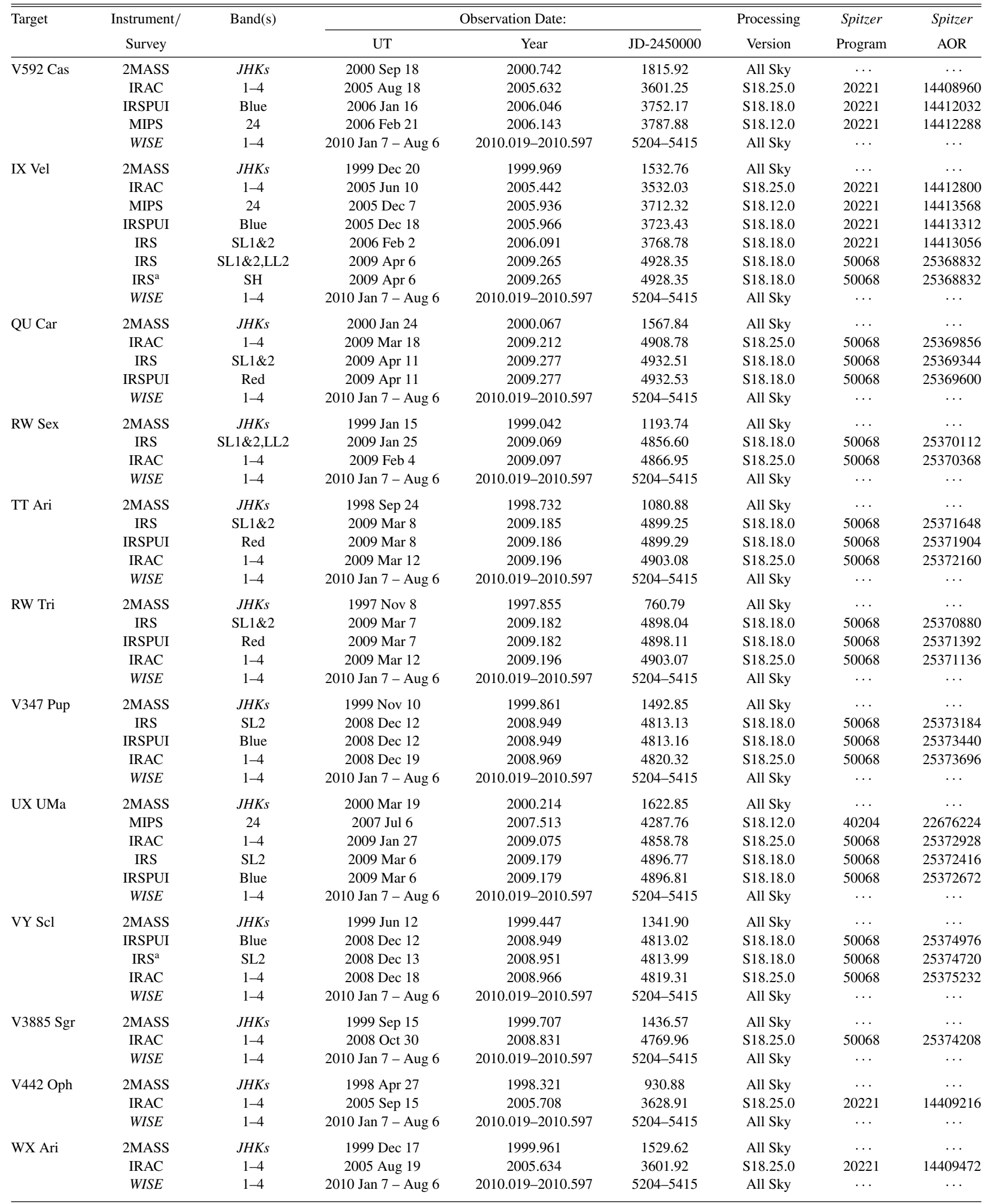

Note. ${ }^{\text {a }}$ These data are not used in this work due to low signal-to-noise ratio. 
Table 3

Infrared Photometry ${ }^{\mathrm{a}}$

\begin{tabular}{|c|c|c|c|c|c|c|c|c|c|c|c|c|c|c|c|c|}
\hline Target & $\begin{array}{c}\text { 2MASS } \\
J \\
1.235\end{array}$ & $\begin{array}{c}\text { 2MASS } \\
H \\
1.662\end{array}$ & $\begin{array}{c}\text { 2MASS } \\
K s \\
2.159\end{array}$ & $\begin{array}{c}\text { WISE } \\
\text { W1 } \\
3.353\end{array}$ & $\begin{array}{c}\text { IRAC } \\
1 \\
3.550\end{array}$ & $\begin{array}{c}\text { IRAC } \\
2 \\
4.493\end{array}$ & $\begin{array}{c}\text { WISE } \\
W 2 \\
4.603\end{array}$ & $\begin{array}{c}\text { IRAC } \\
3 \\
5.731\end{array}$ & $\begin{array}{c}\text { IRAC } \\
4 \\
7.872\end{array}$ & $\begin{array}{c}\text { WISE } \\
\text { W3 } \\
11.561\end{array}$ & $\begin{array}{c}\text { IRSPUI } \\
\text { Blue } \\
15.8\end{array}$ & $\begin{array}{c}\text { WISE } \\
\text { W4 } \\
22.088\end{array}$ & $\begin{array}{c}\text { IRSPUI } \\
\text { Red } \\
22.3\end{array}$ & $\begin{array}{c}\text { MIPS } \\
24 \\
23.68\end{array}$ & Methods ${ }^{\mathrm{b}}$ & Notes \\
\hline V592 Cas & $\begin{array}{c}19.27 \\
\pm 0.53 \\
{[22.28 \pm 1.85]}\end{array}$ & $\begin{array}{c}12.82 \\
\pm 0.44 \\
{[13.95 \pm 1.32]}\end{array}$ & $\begin{array}{c}8.88 \\
\pm 0.25 \\
{[9.35 \pm 0.91]}\end{array}$ & $\begin{array}{r}4.42 \\
\pm 0.12\end{array}$ & $\begin{array}{r}4.15 \\
\pm 0.19\end{array}$ & $\begin{array}{r}2.92 \\
\pm 0.14\end{array}$ & $\begin{array}{r}2.73 \\
\pm 0.07\end{array}$ & $\begin{array}{r}1.98 \\
\pm 0.11\end{array}$ & $\begin{array}{r}1.28 \\
\pm 0.06\end{array}$ & $\begin{array}{c}1.08 \\
\pm 0.08 \\
{[0.74 \pm 0.02]}\end{array}$ & $\begin{array}{r}0.50 \\
\pm 0.04\end{array}$ & $<2.07$ & $\cdots$ & $\begin{array}{r}0.43 \\
\pm 0.03\end{array}$ & I I A & 1,2 \\
\hline IX Vel & $\begin{array}{r}359.16 \\
\pm 12.44\end{array}$ & $\begin{array}{r}261.76 \\
\pm 7.95\end{array}$ & $\begin{array}{l}196.24 \\
\pm 5.06\end{array}$ & $\begin{array}{r}97.70 \\
\pm 2.48\end{array}$ & $\begin{array}{r}90.37 \\
\pm 4.08\end{array}$ & $\begin{array}{r}62.91 \\
\pm 2.83\end{array}$ & $\begin{array}{r}58.53 \\
\pm 1.40\end{array}$ & $\begin{array}{r}42.18 \\
\pm 1.91\end{array}$ & $\begin{array}{r}26.01 \\
\pm 1.18\end{array}$ & $\begin{array}{r}13.77 \\
\pm 0.34\end{array}$ & $\begin{array}{r}7.28 \\
\pm 0.55\end{array}$ & $\begin{array}{r}4.32 \\
\pm 1.08\end{array}$ & $\cdots$ & $\begin{array}{r}3.91 \\
\pm 0.25\end{array}$ & I A A & $\cdots$ \\
\hline QU Car & $\begin{array}{r}65.12 \\
\pm 1.75\end{array}$ & $\begin{array}{r}46.89 \\
\pm 1.29\end{array}$ & $\begin{array}{r}34.77 \\
\pm 0.93\end{array}$ & $\begin{array}{r}19.05 \\
\pm 0.48\end{array}$ & $\begin{array}{r}15.30 \\
\pm 0.77\end{array}$ & $\begin{array}{r}10.72 \\
\pm 0.71\end{array}$ & $\begin{array}{r}11.30 \\
\pm 0.26\end{array}$ & $\begin{array}{r}7.52 \\
\pm 0.40\end{array}$ & $\begin{array}{r}4.84 \\
\pm 0.23\end{array}$ & $\begin{array}{r}2.80 \\
\pm 0.10\end{array}$ & $\cdots$ & $<1.28$ & $\begin{array}{r}0.73 \\
\pm 0.05\end{array}$ & $\cdots$ & I I - & 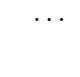 \\
\hline RW Sex & $\begin{array}{l}118.60 \\
\pm 3.54\end{array}$ & $\begin{array}{r}89.60 \\
\pm 2.66\end{array}$ & $\begin{array}{r}62.52 \\
\pm 1.70\end{array}$ & $\begin{array}{r}32.14 \\
\pm 0.86\end{array}$ & $\begin{array}{r}30.13 \\
\pm 1.46\end{array}$ & $\begin{array}{r}20.85 \\
\pm 0.99\end{array}$ & $\begin{array}{r}18.65 \\
\pm 0.44\end{array}$ & $\begin{array}{r}13.81 \\
\pm 0.66\end{array}$ & $\begin{array}{r}8.45 \\
\pm 0.40\end{array}$ & $\begin{array}{r}4.39 \\
\pm 0.17\end{array}$ & $\cdots$ & $<1.94$ & $\cdots$ & $\cdots$ & I - - & $\cdots$ \\
\hline TT Ari & $\begin{array}{r}63.57 \\
\pm 1.71\end{array}$ & $\begin{array}{r}44.37 \\
\pm 1.28\end{array}$ & $\begin{array}{r}29.70 \\
\pm 0.81\end{array}$ & $\begin{array}{r}1.67 \\
\pm 0.04 \\
{[18.2]}\end{array}$ & $\begin{array}{r}15.77 \\
\pm 0.71\end{array}$ & $\begin{array}{r}11.46 \\
\pm 0.52\end{array}$ & $\begin{array}{c}1.18 \\
\pm 0.03 \\
{[9.85]}\end{array}$ & $\begin{array}{r}7.63 \\
\pm 0.34\end{array}$ & $\begin{array}{r}5.10 \\
\pm 0.29\end{array}$ & $\begin{array}{l}<0.40 \\
{[<1.8]}\end{array}$ & $\cdots$ & $\begin{array}{l}<1.83 \\
{[<2.2]}\end{array}$ & $\begin{array}{r}0.90 \\
\pm 0.13\end{array}$ & $\cdots$ & I I - & 3 \\
\hline RW Tri & $\begin{array}{r}26.75 \\
\pm 0.74\end{array}$ & $\begin{array}{r}23.94 \\
\pm 0.71\end{array}$ & $\begin{array}{r}17.38 \\
\pm 0.48\end{array}$ & $\begin{array}{r}7.89 \\
\pm 0.21\end{array}$ & $\begin{array}{r}7.70 \\
\pm 0.35\end{array}$ & $\begin{array}{r}5.53 \\
\pm 0.25\end{array}$ & $\begin{array}{r}4.94 \\
\pm 0.12\end{array}$ & $\begin{array}{r}3.75 \\
\pm 0.17\end{array}$ & $\begin{array}{r}2.59 \\
\pm 0.12\end{array}$ & $\begin{array}{r}1.05 \\
\pm 0.15\end{array}$ & $\cdots$ & $<2.61$ & $\begin{array}{r}0.45 \\
\pm 0.09 \\
{[0.5]}\end{array}$ & $\cdots$ & I I - & 3 \\
\hline V347 Pup & $\begin{array}{r}8.93 \\
\pm 0.27\end{array}$ & $\begin{array}{r}10.42 \\
\pm 0.31\end{array}$ & $\begin{array}{r}8.10 \\
\pm 0.27\end{array}$ & $\begin{array}{r}6.92 \\
\pm 0.18 \\
{[8.1]}\end{array}$ & $\begin{array}{r}7.24 \\
\pm 0.33\end{array}$ & $\begin{array}{r}5.09 \\
\pm 0.23\end{array}$ & $\begin{array}{r}4.08 \\
\pm 0.10 \\
{[4.7]}\end{array}$ & $\begin{array}{r}3.65 \\
\pm 0.17\end{array}$ & $\begin{array}{r}2.36 \\
\pm 0.14\end{array}$ & $\begin{array}{r}0.97 \\
\pm 0.07 \\
{[1.1]}\end{array}$ & $\begin{array}{r}0.76 \\
\pm 0.04\end{array}$ & $\begin{array}{l}<1.73 \\
{[<1.8]}\end{array}$ & $\ldots$ & $\cdots$ & I I - & 3 \\
\hline UX UMa & $\begin{array}{r}12.57 \\
\pm 0.37 \\
{[18.1]}\end{array}$ & $\begin{array}{r}11.13 \\
\pm 0.39 \\
{[14.2]}\end{array}$ & $\begin{array}{r}8.29 \\
\pm 0.25 \\
{[10.1]}\end{array}$ & $\begin{array}{r}5.99 \\
\pm 0.16\end{array}$ & $\begin{array}{r}5.42 \\
\pm 0.25\end{array}$ & $\begin{array}{r}3.88 \\
\pm 0.18\end{array}$ & $\begin{array}{r}3.76 \\
\pm 0.09\end{array}$ & $\begin{array}{r}2.73 \\
\pm 0.12\end{array}$ & $\begin{array}{r}1.69 \\
\pm 0.08\end{array}$ & $\begin{array}{r}0.82 \\
\pm 0.10\end{array}$ & $\begin{array}{r}0.51 \\
\pm 0.02\end{array}$ & $<2.22$ & $\cdots$ & $\begin{array}{r}0.30 \\
\pm 0.04\end{array}$ & I I I & 3 \\
\hline VY Scl & $\begin{array}{l}11.66 \\
\pm 0.31\end{array}$ & $\begin{array}{r}7.84 \\
\pm 0.26\end{array}$ & $\begin{array}{r}5.55 \\
\pm 0.17\end{array}$ & $\begin{array}{r}2.69 \\
\pm 0.07\end{array}$ & $\begin{array}{r}1.03 \\
\pm 0.05 \\
{[2.4]}\end{array}$ & $\begin{array}{r}0.78 \\
\pm 0.04 \\
{[1.6]}\end{array}$ & $\begin{array}{r}1.67 \\
\pm 0.05\end{array}$ & $\begin{array}{r}0.56 \\
\pm 0.03 \\
{[1.1]}\end{array}$ & $\begin{array}{r}0.36 \\
\pm 0.02 \\
{[0.6]}\end{array}$ & $<0.57$ & $\begin{array}{r}0.050 \\
\pm 0.004 \\
{[0.15]}\end{array}$ & $<2.02$ & $\cdots$ & $\cdots$ & I I - & 3 \\
\hline V3885 Sgr & $\begin{array}{r}166.14 \\
\pm 4.96\end{array}$ & $\begin{array}{r}130.71 \\
\pm 4.46\end{array}$ & $\begin{array}{r}94.97 \\
\pm 2.84\end{array}$ & $\begin{array}{r}50.90 \\
\pm 1.41\end{array}$ & $\begin{array}{r}48.29 \\
\pm 2.17\end{array}$ & $\begin{array}{r}34.39 \\
\pm 1.59\end{array}$ & $\begin{array}{r}30.63 \\
\pm 0.78\end{array}$ & $\begin{array}{r}24.43 \\
\pm 1.11\end{array}$ & $\begin{array}{r}15.35 \\
\pm 0.70\end{array}$ & $\begin{array}{r}7.45 \\
\pm 0.24\end{array}$ & $\ldots$ & $<2.95$ & $\cdots$ & $\cdots$ & I - - & $\cdots$ \\
\hline V442 Oph & $\begin{array}{r}7.43 \\
\pm 0.22\end{array}$ & $\begin{array}{r}5.23 \\
\pm 0.20\end{array}$ & $\begin{array}{r}3.78 \\
\pm 0.16\end{array}$ & $\begin{array}{r}0.88 \\
\pm 0.03 \\
{[1.3]}\end{array}$ & $\begin{array}{r}1.15 \\
\pm 0.05\end{array}$ & $\begin{array}{r}0.94 \\
\pm 0.04\end{array}$ & $\begin{array}{c}0.54 \\
\pm 0.03 \\
{[0.75]}\end{array}$ & $\begin{array}{r}0.62 \\
\pm 0.03\end{array}$ & $\begin{array}{r}0.56 \\
\pm 0.05\end{array}$ & $\begin{array}{l}<0.30 \\
{[<0.3]}\end{array}$ & $\cdots$ & $\begin{array}{l}<2.15 \\
{[<2.2]}\end{array}$ & $\cdots$ & $\cdots$ & I - - & 3 \\
\hline WX Ari & $\begin{array}{r}2.74 \\
\pm 0.10\end{array}$ & $\begin{array}{r}2.35 \\
\pm 0.12\end{array}$ & $\begin{array}{r}1.80 \\
\pm 0.09\end{array}$ & $\begin{array}{r}1.02 \\
\pm 0.03\end{array}$ & $\begin{array}{r}1.07 \\
\pm 0.05\end{array}$ & $\begin{array}{r}0.78 \\
\pm 0.04\end{array}$ & $\begin{array}{r}0.70 \\
\pm 0.03\end{array}$ & $\begin{array}{r}0.57 \\
\pm 0.03\end{array}$ & $\begin{array}{r}0.44 \\
\pm 0.03\end{array}$ & $<0.58$ & $\cdots$ & $<2.89$ & $\cdots$ & $\ldots$ & . & \\
\hline
\end{tabular}

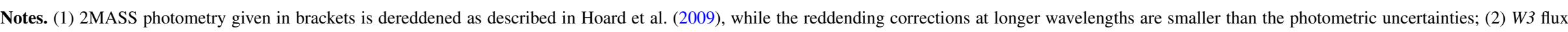
density given in brackets is the PRF-fit value derived in this work; (3) flux density values given in brackets are offset-corrected values discussed in this work.

${ }^{a}$ All flux densities are given in mJy and wavelengths in $\mu \mathrm{m}$.

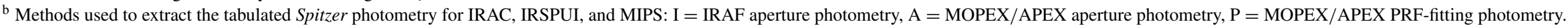




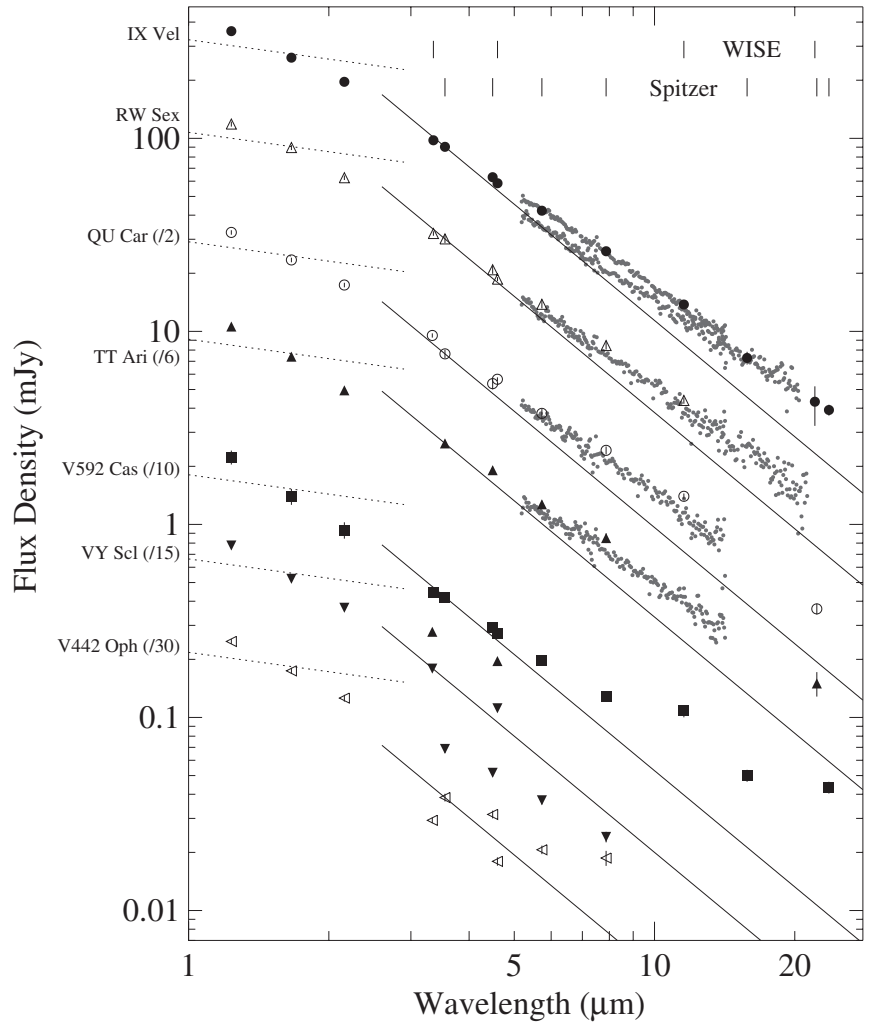

Figure 1. IR SEDs of NLs, constructed from 2MASS, WISE, and Spitzer data. Isophotal wavelengths of the WISE and Spitzer photometric bands are indicated. The Spitzer spectroscopic data are shown as small, gray filled circles. The photometric data are symbol-coded for each CV and, in some cases, have been offset via a constant scale factor for clarity. From top to bottom, the data correspond to IX Vel (filled circles), RW Sex (unfilled upward triangles), QU Car (unfilled circles; scale factor of $1 / 2$ ), TT Ari (filled upward triangles; scale factor of 1/6), V592 Cas (filled squares; scale factor of 1/10), VY Scl (filled downward triangles; scale factor of 1/15), and V442 Oph (unfilled left-pointing triangles; scale factor of 1/30). $1 \sigma$ error bars are plotted on all of the photometric data, but in some cases are smaller than the plot symbols. The dotted lines show the "disk spectrum" portion of the steady state accretion disk SED $\left(f_{v} \propto \lambda^{-1 / 3}\right.$; Frank et al. 2002), normalized to the mean flux density of the three 2MASS bands. The solid lines show the Rayleigh-Jeans tail portion of the accretion disk SED $\left(f_{v} \propto \lambda^{-2}\right.$; Frank et al. 2002), normalized to the Spitzer IRAC-1 $(3.55 \mu \mathrm{m})$ flux density.

our observation epochs. Specific details of corrections applied to data sets on a target-by-target basis are provided below. The corrected data are plotted in Figures 3 and 4 (see discussion in Section 3.1).

\subsection{Data Processing Notes for Individual Targets}

The following sections describe any specific issues that arose during the data processing for each target. In addition, we examined the WISE All Sky Atlas images for each target; except as noted below for V592 Cas and RW Tri, we did not find any anomalous features (either real or due to artifacts) that would affect the cataloged WISE photometry.

\subsubsection{V592 Cas}

For this work, we utilized the final data processing from the Spitzer Heritage Archive ${ }^{24}$ to re-extract the IR photometry for V592 Cas, as well as utilizing the WISE All Sky Survey photometry, which were not available for Hoard et al. (2009). Flux densities are listed in Table 3; for the most part, the new

\footnotetext{
${ }^{24}$ See http://sha.ipac.caltech.edu.
}

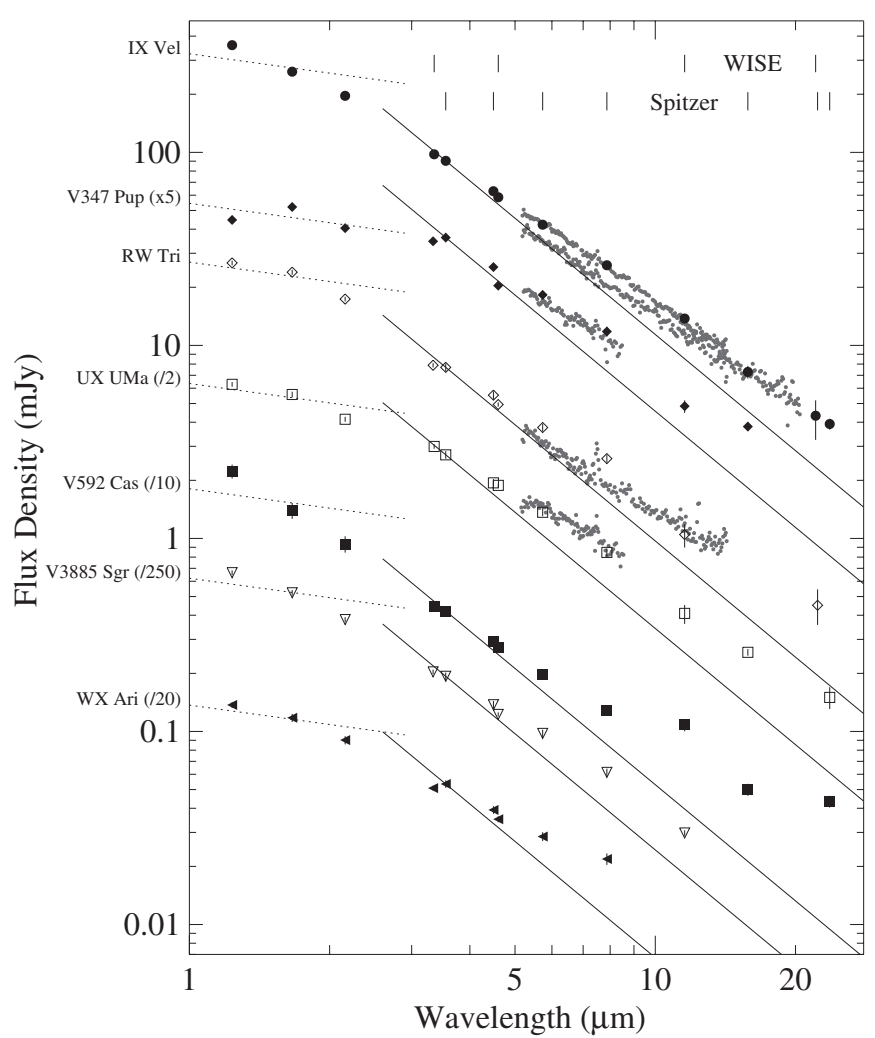

Figure 2. As in Figure 1, for additional NLs. From top to bottom, the data correspond to IX Vel (filled circles), V347 Pup (filled diamonds; scale factor of five), RW Tri (unfilled diamonds), UX UMa (unfilled squares; scale factor of 1/2), V592 Cas (filled squares; scale factor of 1/10), V3885 Sgr (unfilled downward triangles; scale factor of $1 / 250$ ), and WX Ari (filled left-pointing triangles; scale factor of 1/20). IX Vel and V592 Cas are repeated from Figure 1 for comparison.

Spitzer values differ only slightly $(\lesssim 1 \%)$ from those given in Hoard et al. (2009). The exceptions are the IRAC-4 $(7.9 \mu \mathrm{m})$ and IRSPUI $(15.8 \mu \mathrm{m})$ points, which differ by $+5 \%$ and $-10 \%$, respectively. This is consistent with revisions to the final flux calibration and aperture correction values made for the Spitzer end-of-cryo-mission data archive (Carey et al. 2012).

Harrison et al. (2013) independently re-derived the Spitzer photometry for V592 Cas as part of their examination of the Spitzer photometry and Herschel Space Observatory upper limits for several CVs. They focused on magnetic CVs and DNe, and only V592 Cas is in common between their target sample and ours. They obtained essentially the same photometric values as ours, and also noted the $\approx 10 \%$ decrease in the revised IRSPUI flux density measurement.

The cataloged WISE All Sky W3 $(11.6 \mu \mathrm{m})$ flux density for V592 Cas (1.08 $\pm 0.08 \mathrm{mJy})$ is anomalously bright compared to the overall trend in its SED (see Figures 1 and 2). If real, this would be an important finding, since it could indicate the presence of the $10 \mu \mathrm{m}$ silicate emission feature, which is a hallmark of circumstellar dust around isolated WD stars (Jura et al. 2007, 2009; Reach et al. 2009) and is also observed due to dust in the ejecta of post-outburst classical and recurrent novae (Evans et al. 2007). Such a feature is not seen in the IRS spectra of our other targets; unfortunately, V592 Cas has no IRS spectral observation with which to verify its absence.

The W3 photometry could be contaminated by the brighter neighbor (designated WISE J002053.73+554219.3) that is located 13" east of V592 Cas. Harrison et al. (2013) suggest that 


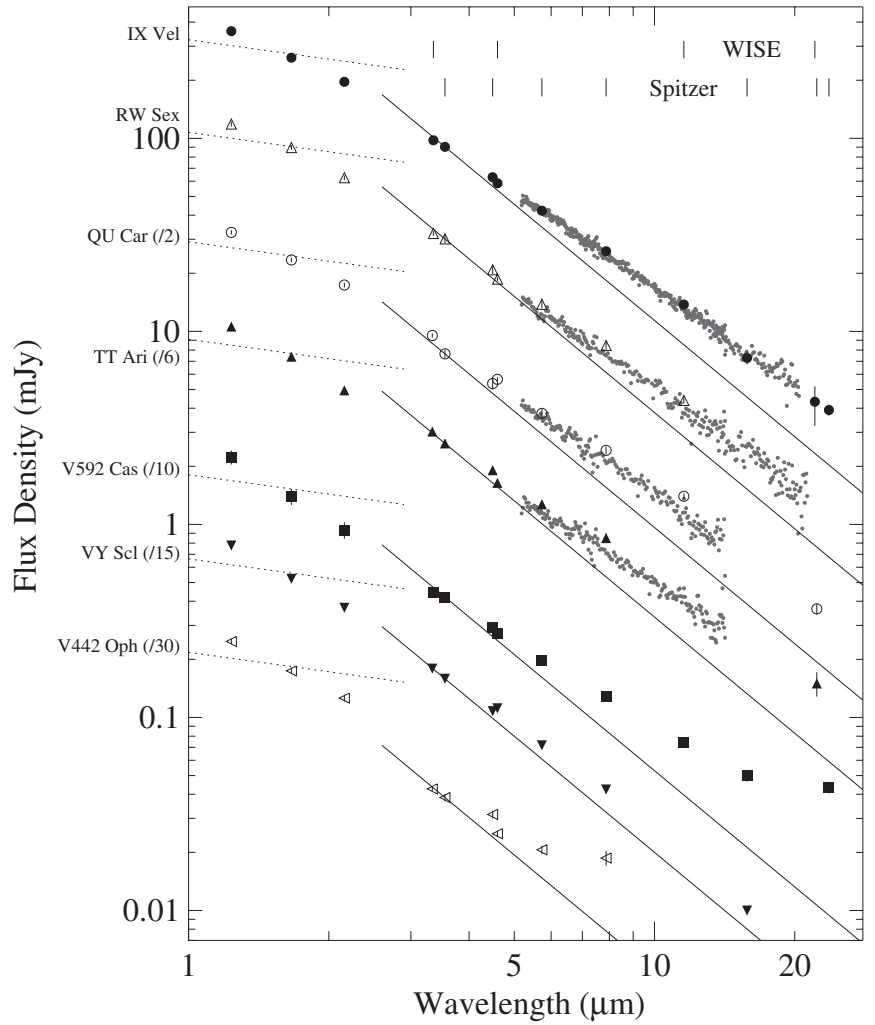

Figure 3. As in Figure 1, but using the RJL-offset-corrected data where applicable (see Section 2.1 and individual target notes in Section 2.2) and the PSF-fit photometry value for the W3 point of V592 Cas (see Section 2.2.1).

the large FWHM of the W3 point-spread function (PSF) ensured that the W3 photometry of V592 Cas was contaminated. Although this explanation is ultimately correct in its conclusion (see below), it is not entirely accurate: the WISE W1 and W2 photometry of V592 Cas agrees closely with the adjacent IRAC1 and IRAC-2 points, so do not appear to be contaminated by the neighboring source, despite the fact that the W1 and W2 PSF FWHMs $(6$ ". $1 \times 5$ ". 6 and 6". $8 \times 6$ ". 1 , respectively) are comparable in size to that of $W 3\left(7^{\prime \prime} .4 \times 6\right.$ ". 1$){ }^{25}$ The crucial factor is that the wings of the $W 3$ PSF are brighter relative to the peak at larger distances from the center compared to the W1 and W2 PSFs; at $13^{\prime \prime}$ from the center, the W3 PSF wings are $\sim 5 \%$ of the peak versus $\sim 1 \%$ of the peak at the same location in the $W 1$ and $W 2$ PSFs.

Given the potential importance of an elevated $W 3$ flux density in V592 Cas, we thoroughly examined the possibility of contamination from the neighboring source. We downloaded the WISE All Sky Release Atlas images of the V592 Cas field from the Infrared Science Archive (IRSA). ${ }^{26}$ We used the bright isolated nearby star WISE J002104.75+554356.9 $(W 2=$ $8.22 \pm 0.02 \mathrm{mag}, W 3=8.12 \pm 0.02 \mathrm{mag}$ ) to define a local PSF, then used the IRAF implementation of the DAOPHOT package (Stetson 1987) to extract PSF-fit photometry for V592 Cas and its nearby neighbor from the $W 2$ and $W 3$ Atlas images. Figure 5 shows the W3 Atlas image of V592 Cas, along with the PSF subtractions of the bright neighbor only and both stars together.

The WISE All Sky Survey catalog photometry for the neighbor is $W 2=9.80(2) \mathrm{mag}$ and $W 3=9.59(3) \mathrm{mag}$; for $\mathrm{V} 592$

\footnotetext{
25 See http://wise2.ipac.caltech.edu/docs/release/allsky/expsup/sec4 4c.html\#coadd_psf.

26 See http://irsa.ipac.caltech.edu/index.html.
}

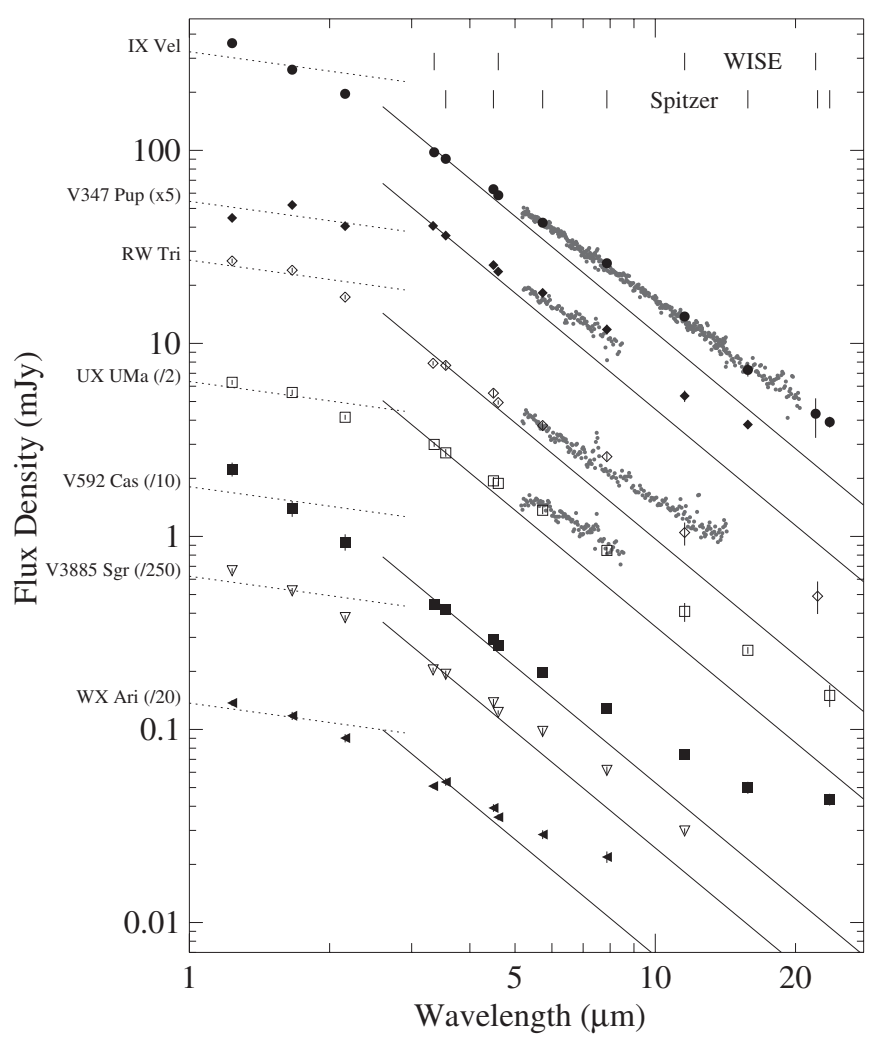

Figure 4. As in Figure 2, but using the RJL-offset-corrected data where applicable (see Section 2.1 and individual target notes in Section 2.2) and the PSF-fit photometry value for the W3 point of V592 Cas (see Section 2.2.1).

Cas, the corresponding photometry is $W 2=12.00(2)$ mag and $W 3=11.17(8)$ mag. We obtain values of $\mathrm{W} 2=9.83(3) \mathrm{mag}$ and $W 3=9.64(3)$ mag for the neighbor, in agreement with the WISE All Sky Survey values (within 1 $\sigma$ ). For V592 Cas, we obtain $W 2=11.99(3)$ mag and $W 3=11.58(3)$ mag; although our $W 2$ value is in agreement with the WISE All Sky Survey value, our $W 3$ value is significantly fainter, corresponding to a flux density of $0.74(2) \mathrm{mJy}$. This value is consistent with the predicted value based on a full SED model (see Section 3.3.1) and is used throughout the rest of this work (e.g., see Figures 3 and 4). So, unfortunately, it appears that there is no enhanced W3band emission in V592 Cas. The target with the next brightest W3 flux density is QU Car (see Section 2.2.3), but our IRS spectrum of this target rules out the presence of the $10 \mu \mathrm{m}$ silicate emission feature. See Section 4.1.1 for additional discussion of the "missing" silicate emission feature in CVs.

\subsubsection{Vel}

Our Spitzer Cycle-5 IRS spectrum of IX Vel is fainter than the Cycle-2 IRS spectrum and IRAC photometry. Unfortunately, we found no useful published or AAVSO brightness state data to correlate with the timing of the Spitzer observations. This would have enabled us to independently estimate a relative offset between the two IRS spectra. Instead, to compensate for the difference in brightness between the two spectra, we added a RJL offset (as described in Section 2.1) to the Cycle-5 spectrum, with initial value of $+8.2 \mathrm{mJy}$ at the short wavelength $(5.2 \mu \mathrm{m})$ end of the Cycle-5 spectrum. This brings the Cycle-5 spectrum into agreement with the Cycle-2 spectrum. There is also a Cycle-5 IRS Short-High (high resolution) spectrum of IX Vel, which is not used here. We note that this spectrum matches the continuum shape of the corresponding IRS low resolution 
spectra, but is considerably noisier and does not show strong emission or absorption features.

\subsection{3. $Q U C a r$}

Observations from the AAVSO and Kafka et al. (2012) show that QU Car was variable between $V \approx 11.2-11.8$ mag during our Spitzer observations. Although the IRS spectrum is slightly faint compared to the Spitzer and WISE photometry, there are no significant discrepancies between the various data sets.

\subsection{4. $R W$ Sex}

The long-term AAVSO light curve for RW Sex shows no significant changes in mean brightness level between the observation dates of the 2MASS, Spitzer, and WISE data sets.

\subsubsection{TT Ari}

The AAVSO light curve of TT Ari shows no significant optical brightness change between the 2MASS and Spitzer observations; however, the WISE photometry was obtained during a faint state between JD 2455100-2455600. During this time, TT Ari was at $V \sim 15.5 \mathrm{mag}$ instead of its normal $V \sim 10.75$ mag. To compensate, we applied a RJL offset (as described in Section 2.1) to the WISE photometry, with initial value of $+16.5 \mathrm{mJy}$ at $\mathrm{W} 1(3.35 \mu \mathrm{m})$. Unlike the case of IX Vel, for which we had a second, bright spectrum in an overlapping wavelength interval with which to estimate the correct offset function for the faint spectrum, we do not have photometry at exactly overlapping wavelengths to compare for TT Ari. Instead, we set the initial value of the offset function such that the corrected WISE photometry falls along the Rayleigh-Jeans tail accretion disk spectrum normalized to the IRAC-1 value (see Section 3.1). Although there is some concern that the SED might manifest a significant non-RJL secondary star contribution during such a deep faint state, the RJL offset does not appear to introduce a significantly discrepant slope to the corrected WISE photometry (see Figure 3).

\subsection{6. $R W T r i$}

The long-term AAVSO light curve of RW Tri shows that there are no significant changes in the mean optical brightness level between the dates of the 2MASS, Spitzer, and WISE observations. However, the IRS spectrum is fainter than the overlapping IRAC-3 and IRAC-4 points. To compensate, we applied a RJL offset (as described in Section 2.1) to the IRS and IRSPUI data (the latter were obtained contemporaneously with the former), with initial value of $+0.7 \mathrm{mJy}$ at $5.2 \mu \mathrm{m}$. The corrected IRS data are in better overall agreement with the other data, albeit still slightly fainter than IRAC-4 and slightly brighter than W3. A faint, linear diffraction artifact passes near RW Tri in the WISE W3 image; however, the surface brightness of the artifact is $\lesssim 5 \%$ of the local background. Consequently, it does not appear to have had any significant effect on the corresponding photometry.

\subsubsection{V347 Pup}

The AAVSO light curve coverage of V347 Pup stops in late 2002; prior data show variability of $\sim 1$ mag (full amplitude) on timescales of hundreds of days, around an approximately constant mean brightness ( $V \sim 13.5 \mathrm{mag}$ ). The WISE photometry is systematically fainter than the Spitzer data. To compensate, we applied a RJL offset (as described in Section 2.1) to the WISE photometry, with initial value of $+1.2 \mathrm{mJy}$ at $\mathrm{W} 1(3.35 \mu \mathrm{m})$. The

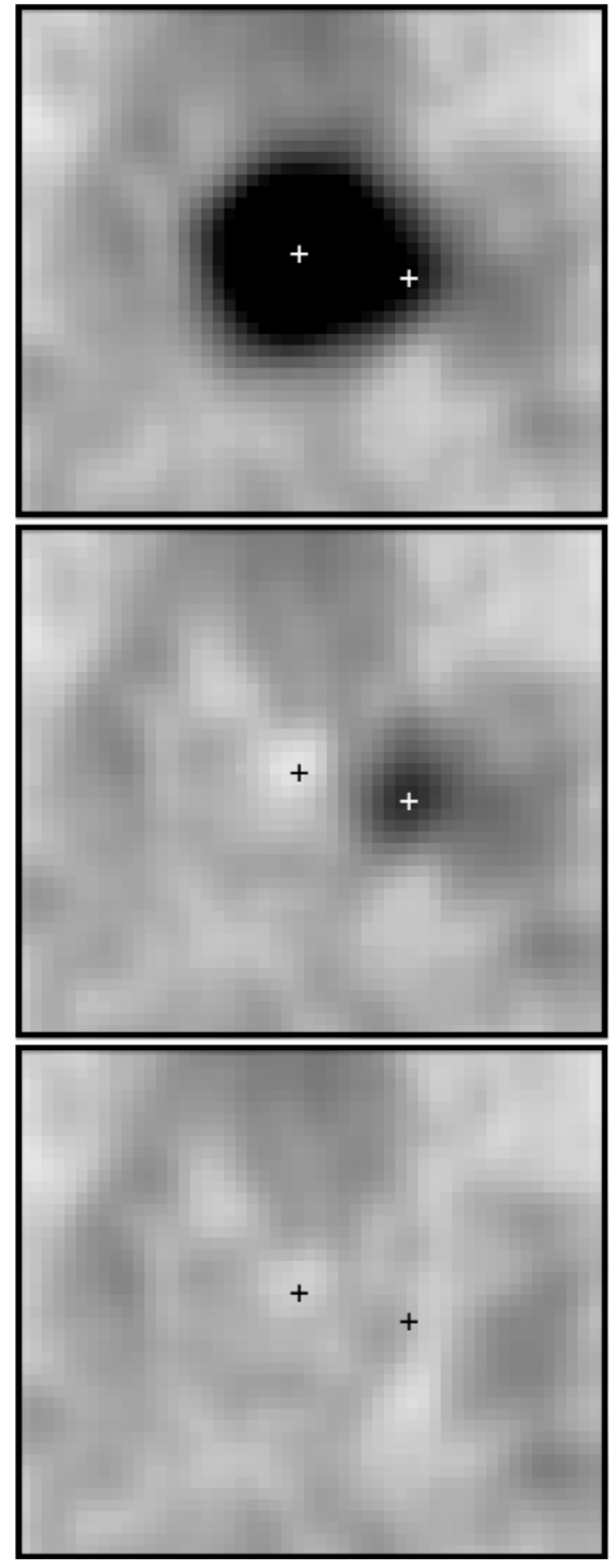

Figure 5. WISE All Sky Release Atlas image of V592 Cas in the W3 band showing the overlapping profiles of V592 Cas and its bright neighbor (top panel). The image is centered on the neighbor. The middle panel shows the image after PSF-subtraction of the bright neighbor star, with only V592 Cas remaining. The bottom panel shows the image after PSF-subtraction of both stars. All panels have a width of $72^{\prime \prime}$, plate scale of $1^{\prime \prime} .375 \mathrm{px}^{-1}$, and identical grayscale stretch. Plus symbols in all of the panels mark the coordinates of the neighbor and V592 Cas from 2MASS and Downes et al. (2001), respectively.

2MASS photometry also seems faint compared to the Spitzer data, but we have no corroborating data with which to determine an appropriate offset. 


\subsubsection{UX UMa}

The AAVSO data for UX UMa show no significant changes in mean brightness level between the observation dates of the 2MASS, Spitzer, and WISE data sets.

\subsection{9. $\mathrm{VY} \mathrm{Scl}$}

The 2MASS observations of VY Scl were obtained during a normal bright state $(V \approx 13.5 \mathrm{mag}$ ). Recent photometric coverage is sparse, but the WISE photometry were likely also obtained during a normal bright state, as they appear to be consistent with the extrapolation in wavelength of the 2MASS values. However, the Spitzer Cycle-5 observations were obtained during the transition from a low state $(V \sim 18 \mathrm{mag})$ back to the bright state. Using the optical light curves in Greiner et al. (2010) and from the AAVSO, we estimate $V \sim 15.5 \mathrm{mag}$ during the IRS observations and $V \sim 14.6 \mathrm{mag}$ during the IRAC observations. To compensate, we applied a RJL offset (as described in Section 2.1) to the IRAC photometry, with initial value of $+1.35 \mathrm{mJy}$ at IRAC-1 $(3.55 \mu \mathrm{m})$. The IRSPUI point, which was obtained when VY Scl was $\Delta V \sim 0.9$ mag fainter than during the IRAC observations, was offset by $+0.1 \mathrm{mJy}$, corresponding to an RJL offset with initial value of $+1.9 \mathrm{mJy}$ at IRAC-1. The Cycle-5 IRS spectrum of VY Scl is very noisy, and is not used or plotted here, although we note that it is roughly consistent with the other Spitzer photometry.

\subsubsection{0. $\mathrm{V3885} \mathrm{Sgr}$}

The AAVSO long-term light curve of V3885 Sgr is stable through the end of coverage in late 2005. The assembled SED shows no obvious signs of flux density level offsets between the various data sets.

\subsubsection{V442 Oph}

Ballouz \& Sion (2009) summarize the optical variability history of V442 Oph, which ranges from $V=12.6 \mathrm{mag}$ to $V=15.5 \mathrm{mag}$, although the typical faint state level is $V=14.0$ mag. Unfortunately, the AAVSO coverage of V442 Oph is very sparse, with only a handful of validated data points in the past two decades. The WISE data are fainter than the corresponding IRAC photometry. To compensate, we applied a RJL offset (as described in Section 2.1) to the WISE photometry, with initial value of $+0.4 \mathrm{mJy}$ at $\mathrm{W} 1(3.35 \mu \mathrm{m})$. The offset W2 flux density is still slightly fainter than IRAC-2.

\subsubsection{WX Ari}

There are no AAVSO data for WX Ari during 1999-2009. It was in a faint state ( $V \sim 17.5 \mathrm{mag}$ ) below its normal brightness level ( $V \sim 15 \mathrm{mag}$ ) from late 2012 until early 2013, but the decline to this state does not appear to have started until late2011 and would not have affected any of the data used here.

\section{ANALYSIS}

In this section we will establish that the IR SEDs of these NLs deviate from the expectation of the standard steady state accretion disk theory (Section 3.1), correlate the strength of this deviation with two fundamental system parameters (inclination and orbital period; Section 3.2), and construct physically realistic, multi-component models to reproduce the observed SEDs of several of our targets (Section 3.3).

\subsection{Infrared Excess}

Frank et al. (2002) review the theoretical spectrum of a steady state $\mathrm{CV}$ accretion disk, and divide it into regions of the Wien exponential, which is dominated by the hottest inner regions of the disk $\left(T \sim T_{\mathrm{wd}}\right)$, the "characteristic disk spectrum" at intermediate temperatures, which has $f_{v} \propto \lambda^{-1 / 3}$, and the Rayleigh-Jeans tail for the coolest disk regions, which is characterized by $f_{v} \propto \lambda^{-2}$. NLs, in particular, are expected to have accretion disks that conform to this prescription, although it has been known for some time that the observed NL disk spectra tend to be redder than predicted by the standard model at short wavelengths corresponding to UV emission from the hot, inner disk (e.g., Wade 1988). This is often interpreted as evidence for a truncation of the inner disk-so that the expected hottest regions are absent and do not contribute to the observed spectrum-but the exact reason for the discrepancy is currently uncertain.

We are focusing here on the IR wavelengths corresponding to the cooler, outer accretion disk. Before moving on to consideration of the mid-IR region of the SEDs, we first fit a characteristic disk spectrum to just the near-IR 2MASS data for each of our targets, normalized to the mean flux density of the three bands. For many of the targets, the JHKs data points are linearly decreasing (in log-log space), and have a steeper (negative) slope than the characteristic disk spectrum model, implying that the actual $f_{v} \propto \lambda^{-1 / 3}$ region occurs at shorter wavelengths, and the near-IR is already transitioning into the Rayleigh-Jeans tail spectrum. Szkody (1977) noticed a similar trend in pioneering near-IR $(\mathrm{JHK})$ observations of six DNe obtained at or near maximum light, along with TT Ari (whose nature at the time was uncertain but believed to be related to the $\mathrm{DNe}$ ). The targets displaying this behavior in our investigation are IX Vel, RW Sex, QU Car, TT Ari, V592 Cas, VY Scl, and V442 Oph (i.e., the targets shown in Figures 1 and 3 ).

The remaining targets, V347 Pup, RW Tri, UX UMa, V3885 Sgr, and WX Ari (i.e., the targets shown in Figures 2 and 4) have "broken" near-IR flux densities; that is, the J-to-H slope is different from the $H$-to- $K s$ slope (in the case of V347 Pup, the two slopes even have opposite signs). In these five targets, there is generally better agreement with the characteristic disk spectrum prediction, especially with the $J$ and $H$ points. On the other hand, the $H$-to- $K s$ slope is steeper than the $f_{\nu} \propto \lambda^{-1 / 3}$ prediction for all of the targets, suggesting that the transition to the Rayleigh-Jeans tail portion of the steady state accretion disk spectrum is occurring longward of $H$ band.

Harrison et al. $(2004,2005)$ identified features from the secondary star in $K$-band spectra of most of a sample of two dozen CVs (primarily DNe) spanning a wide range of orbital period (1.9-11 hr). They found that, unsurprisingly, the brighter, early type secondary stars in long period CVs are more easily detected, but also that the contribution from a bright accretion disk can make the identification and spectral typing of the secondary star difficult. This situation is exacerbated among the NLs, in which the generally high mass transfer rates produce prominent disks that can dominate the SEDs over a wide range of wavelengths. Dhillon et al. (2000) found that in a sample of six bright NLs (including RW Tri and VY Scl), none show evidence of the secondary star in their $K$-band spectra. Nonetheless, we must consider that the secondary star could make a nonnegligible contribution to the observed near-IR SED for some of our targets, which would produce disagreement with the Frank et al. (2002) model accretion disk spectrum. For example, while 
the secondary star in V592 Cas contributes only $\approx 3 \%$ of the total light at $H$ band (see SED models in Hoard et al. 2009 and Section 3.3.1), the secondary star contribution at $H$ band in IX Vel is $\approx 17 \%$ (see SED model in Section 3.3.2). V347 Pup has a larger, hotter secondary star (M0.5; Thoroughgood et al. $2005)$ and smaller mass transfer rate $\left(6 \times 10^{-9} M_{\odot} \mathrm{yr}^{-1}\right.$; Puebla et al. 2007) than V592 Cas (M4.1, $1.5 \times 10^{-8} M_{\odot} \mathrm{yr}^{-1}$; see below), so likely has more secondary star "contamination" of the disk spectrum in the near-IR. Similarly, in UX UMa, which has a relatively low mass transfer rate of $8 \times 10^{-9} M_{\odot} \mathrm{yr}^{-1}$ (see below), the M2.6 secondary star contributes $\approx 40 \%$ of the total light at $H$ band (see Section 3.3.3).

We fit the longer wavelength Spitzer and WISE data with a Rayleigh-Jeans tail spectrum, normalized to the IRAC-1 $(3.55 \mu \mathrm{m})$ flux density point for each target. In all cases, the observed data show IR SEDs at $\lambda>3.55 \mu \mathrm{m}$ with continuum slopes shallower than $f_{v} \propto \lambda^{-2}$; hence, their SEDs show an excess of flux density relative to the expectation of the standard steady state accretion disk theory. This IR excess is apparent at wavelengths as short as $\approx 4.5 \mu \mathrm{m}$ (IRAC-2, W2) and increases in strength at longer wavelengths. Clearly, there is a "nonstandard" contribution to the IR SED in all of these CVs.

A pertinent question is whether the observed IR excess is due to a shortcoming in the standard model treatment or the presence of an additional system component. The former option presumes that the spectrum of an accretion disk in the IR might not be well-represented by a sum of blackbodies. There has been little work in detailed model atmosphere synthetic spectrum modeling of $\mathrm{CV}$ accretion disks in the IR (with most work to date focussing on the ultraviolet spectral region). Linnell et al. $(2007 \mathrm{a}, 2007 \mathrm{~b})$ have shown that from the optical out to $\approx 3.5 \mu \mathrm{m}$, the SED continuum shape returned by disk model atmosphere calculations still conforms to the expectation from the blackbody-based steady state theory described in Frank et al. (2002). It remains to be seen whether the detailed physics of very cool gas contributing at even longer wavelengths could produce the observed SEDs. We concentrate here on the latter option, in which the observed IR excess is due to the presence of a previously unconsidered physical component of the CV.

In Figure 6, we have replotted the SED data from Figures 3 and 4, but have normalized the Spitzer and WISE data by dividing by the corresponding Rayleigh-Jeans tail accretion disk model shown in Figures 3 and 4, which was, in itself, normalized to the Spitzer IRAC-1 flux density. The spectroscopic data only are then renormalized by multiplying by the ratio of the IRAC$3(5.7 \mu \mathrm{m})$ flux density to the mean of each spectrum in the wavelength range 5.4-6 $\mu \mathrm{m}$. Thus, the horizontal dotted line at a constant normalized flux value of 1.0 in the figure shows the Rayleigh-Jeans disk model SED ( $f_{v} \propto \lambda^{-\alpha}$, with $\left.\alpha=2\right)$, and the IR excess of each target relative to this model is readily apparent. Additional dotted lines in the figure show shallower spectral profiles with smaller values of the index $\alpha$.

We note that there is no special physical significance to this (re)normalization process: it simply provides a means of easily comparing the IR excess in multiple CVs that have different distances and intrinsic luminosities. The choice of the IRAC-1 point for normalizing the theoretical accretion disk spectrum is motivated primarily by the desire to choose a wavelength long enough that the "standard" CV components are on the Rayleigh-Jeans tail, but short enough that any "non-standard" components make a negligibly small contribution. As described above, there are obvious deviations in the JHKs bands from the Rayleigh-Jeans tail expectation of the steady state accretion

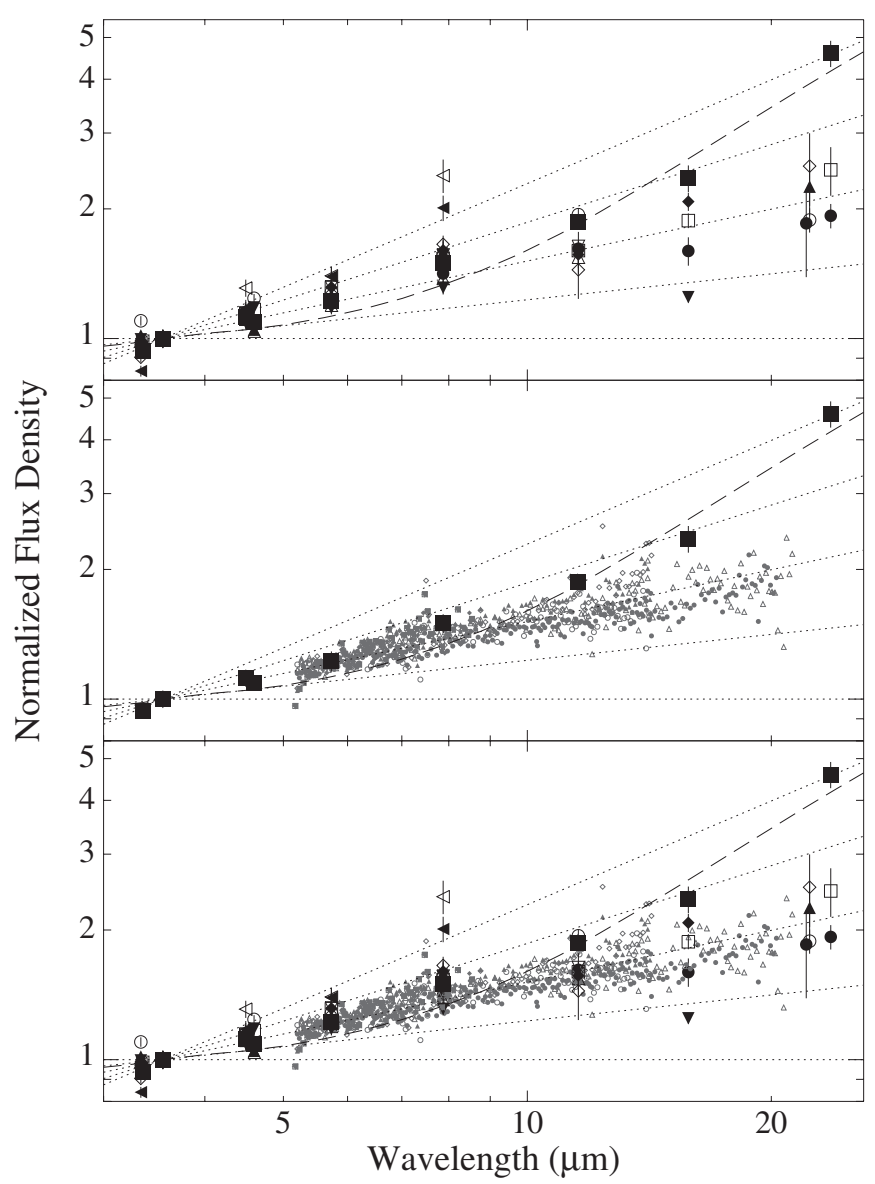

Figure 6. Normalized SEDs showing the data from Figures 3 and 4 (with the same symbol coding), but normalized to the Rayleigh-Jeans model accretion disk SEDs (horizontal dotted line). An ordinate value of $y$ indicates that the observed flux density is a factor of $y$ larger than the value expected at that wavelength for the Rayleigh-Jeans disk model alone. The top panel shows only the photometric data, the middle panel shows only the spectroscopic data, and the bottom panel shows all of the data. For comparison, the V592 Cas photometric data (large filled squares) and system model (dashed line; see Section 3.3.1) are shown in all of the panels. The dotted lines show normalized spectral profiles of $f_{v} \propto \lambda^{-\alpha}$, where $\alpha=2.0,1.9,1.8,1.7$, and 1.6 , respectively from bottom to top.

disk theory for many of our targets. The shortest wavelength data available longward of $K s$ band are from the $W 1$ and IRAC- 1 bands at $\approx 3.5 \mu \mathrm{m}$; the latter is preferred over the former because the IRAC-1 photometry has smaller uncertainties than $W 1$. Hindsight from the multi-component model fits confirms that the SEDs at $3.55 \mu \mathrm{m}$ contain only a very small non-standard (IR excess) component but a large standard (accretion disk + secondary star) component (see Section 3.3).

There are two features of note in Figure 6:

First, V592 Cas has the most prominent long-wavelength $(\lambda \gtrsim 15 \mu \mathrm{m})$ IR excess of any of these targets. At $7.9 \mu \mathrm{m}$ (IRAC-4), only V442 Oph and WX Ari have an IR excess larger than that of V592 Cas. These two CVs are also both brighter than V592 Cas (relative to the disk model) at the shorter IRAC-2 $(4.5 \mu \mathrm{m})$ and IRAC-3 $(5.6 \mu \mathrm{m})$ wavelengths, although to a lesser extent than at IRAC-4 $(7.9 \mu \mathrm{m})$, where they are the brightest $\mathrm{CVs}$ in our sample relative to the disk model. However, IRAC-4 is the longest wavelength photometric point currently available for WX Ari and V442 Oph, so it is uncertain if their IR excesses are significantly stronger than that of V592 Cas all the way to $24 \mu \mathrm{m}$. 


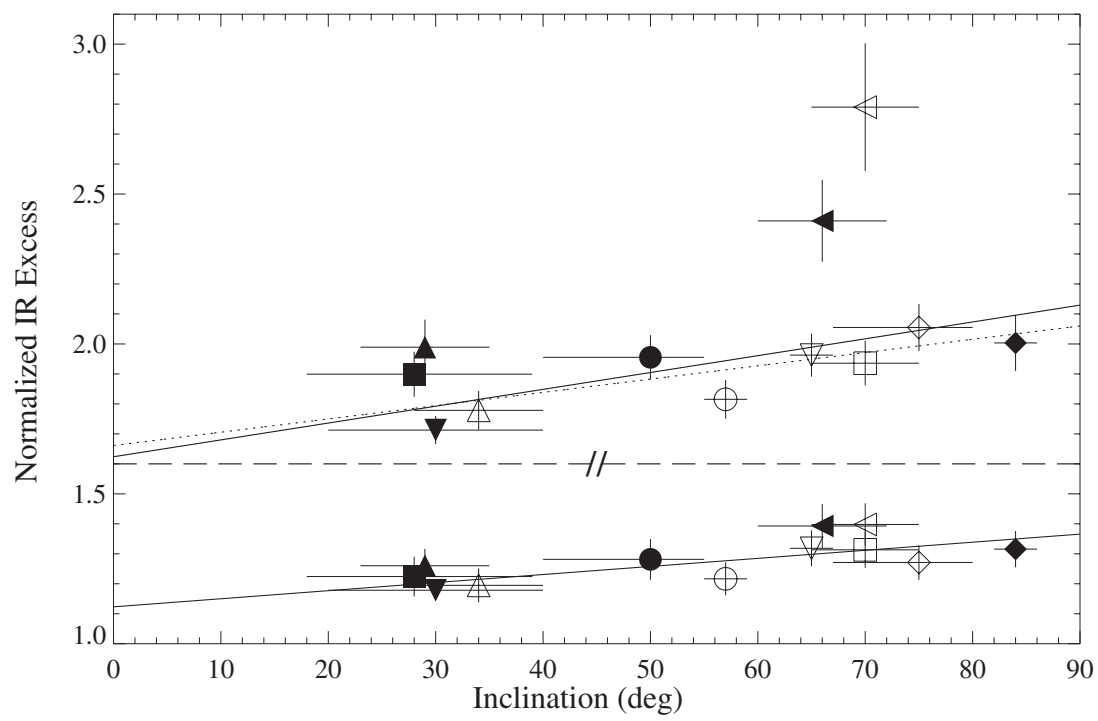

Figure 7. Relation between system inclination (see Table 1) and normalized IR excess (from Figure 6). The lower data show the IRAC-3 (5.7 $\mu$ m) excess, while the upper data (separated by the broken dashed line) show the IRAC-4 $(7.9 \mu \mathrm{m})$ excess (offset by +0.4 for clarity). The solid lines are linear fits to each data set, weighted by the uncertainties on both parameters. The dotted line is the linear fit to the $7.9 \mu \mathrm{m}$ data after excluding the two outlying points. Plot symbols are coded by target as in Figures 3, 4, and 6.

Table 4

System Parameter Correlations

\begin{tabular}{|c|c|c|c|c|c|c|}
\hline \multirow[t]{2}{*}{ Case $^{\mathrm{a}}$} & \multirow[t]{2}{*}{ Parameter } & \multirow{2}{*}{$\begin{array}{c}\lambda_{\text {excess }} \\
(\mu \mathrm{m}) \\
\end{array}$} & \multicolumn{2}{|c|}{ Linear Fit: } & \multicolumn{2}{|c|}{ Spearman's Rank: } \\
\hline & & & Slope & $\tilde{\chi}^{2}$ & $\rho$ & $P^{\mathrm{b}}$ \\
\hline \multirow[t]{4}{*}{ A } & Inclination & 5.7 & $0.0027(8) \mathrm{deg}^{-1}$ & 0.8 & 0.65 & 0.02 \\
\hline & & 7.9 & $0.0056(13) \mathrm{deg}^{-1}$ & 3.9 & 0.62 & 0.03 \\
\hline & Orbital period & 5.7 & $-0.22(22) \mathrm{d}^{-1}$ & 2.1 & -0.29 & 0.35 \\
\hline & & 7.9 & $-0.26(27) \mathrm{d}^{-1}$ & 6.6 & -0.36 & 0.25 \\
\hline \multirow[t]{4}{*}{ B } & Inclination & 5.7 & $0.0022(9) \mathrm{deg}^{-1}$ & 0.5 & 0.65 & 0.04 \\
\hline & & 7.9 & $0.0044(13) \mathrm{deg}^{-1}$ & 1.7 & 0.55 & 0.10 \\
\hline & Orbital period & 5.7 & $0.03(23) \mathrm{d}^{-1}$ & 1.6 & -0.03 & 0.93 \\
\hline & & 7.9 & $0.07(27) \mathrm{d}^{-1}$ & 3.9 & -0.12 & 0.75 \\
\hline
\end{tabular}

Notes.

${ }^{a}$ Case A includes all of the targets, while case B excludes the outliers WX Ari and V442 Oph.

${ }^{\mathrm{b}} P$ is the significance of the correlation (i.e., the probability that it could have occurred by chance).

Second, across the entire wavelength range of the data, but especially at $\lambda \lesssim 10 \mu \mathrm{m}$, the normalized photometry and spectroscopy for each target are remarkably similar. With the exceptions of V592 Cas, WX Ari, and V442 Oph, the targets in our sample have IR SEDs consistent with a spectral index of $\alpha \approx 1.8 \pm 0.1$ in the wavelength range $\Delta \lambda \approx 3.5-25 \mu \mathrm{m}$. This implies that the spectral profile of the component producing the IR excess in these targets is similar. The IR SEDs of WX Ari and V442 Oph are consistent with a shallower spectral index of $\alpha \lesssim 1.6$ out to their last available data points at $7.9 \mu \mathrm{m}$, while that of V592 Oph does not follow a simple power law over the entire wavelength range.

\subsection{Correlations with System Parameters}

In Figure 7, we show the relation between system inclination (from Table 1) and IR excess at 5.7 and $7.9 \mu \mathrm{m}$ (the normalized IRAC-3 and IRAC-4 values from Figure 6). Linear fits to each data set, weighted by the uncertainties of both parameters, reveal shallow slopes of increasing IR excess with increasing inclination. The fit parameters are listed in Table 4. There is little change in the linear fit results when the two outliers in the $7.9 \mu \mathrm{m}$ excess data set, WX Ari and V442 Oph, are excluded from both data sets. As discussed above, these two targets-along with V592 Cas - have the strongest IR excesses in our sample. V442 Oph and WX Ari would benefit from additional long wavelength observations at $\lambda>8 \mu \mathrm{m}$, since their IR excess appears to still be growing stronger at the longest available wavelength in their SEDs (IRAC-4). We also calculated the Spearman's rank correlation coefficient, $\rho$, for these two cases (see Table 4). This allows us to make a more general assessment of whether these two parameters are related by a monotonic function of any form (not necessarily linear). The results are consistent with those of the linear fits: there is a strong, statistically significant, positive correlation between inclination and IR excess at both wavelengths, regardless of whether or not the two outliers are excluded. The probability of these correlations occurring by chance is small ( $\leqslant 10 \%$ in all cases, and $<5 \%$ in three of the four cases).

It is tempting to ascribe the shallow trend of increasing IR excess with increasing inclination to an increasing visibility of the relatively cool accretion disk rim (or, conversely, decreasing visibility of the hot inner disk) with increasing inclination. The observed change in normalized IR excess at $7.9 \mu \mathrm{m}$ going 


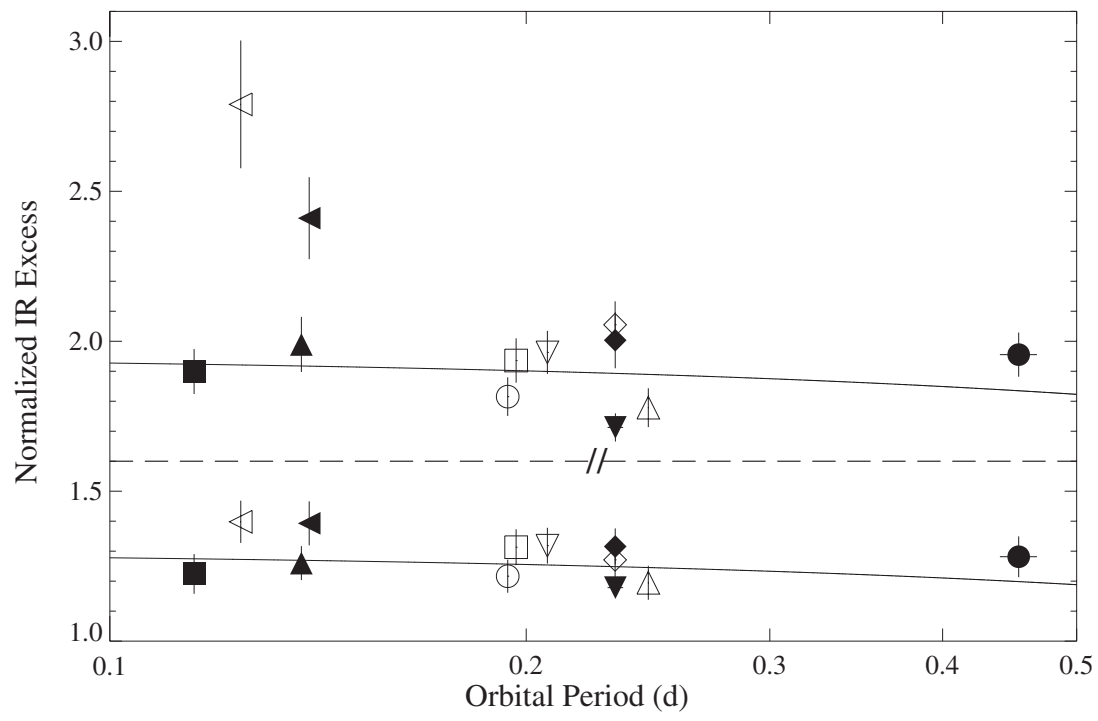

Figure 8. As in Figure 7, but for orbital period (see Table 1) and normalized IR excess.

from $i \approx 30^{\circ}$ (when little accretion disk rim is directly visible) to $i \approx 90^{\circ}$ (when only the disk rim is visible) amounts to an increase of $\approx 20 \%$. The SED of a model steady state accretion disk using the parameters for V592 Cas $\left(i=28^{\circ}\right.$; see Sections 3.3 and 3.3.1) is significantly fainter when recalculated for an inclination of $90^{\circ}$ (owing to the substantial decrease in overall projected emitting surface area). The corresponding increase in normalized IR excess relative to $3.55 \mu \mathrm{m}$ (IRAC-1) at $7.87 \mu \mathrm{m}$ (IRAC-4) or $23.68 \mu \mathrm{m}$ (MIPS-24) is only $\approx 4 \%$ or $\approx 6.5 \%$ (owing to the occultation of the hot inner disk and increased view of the cooler disk rim). This argues against the accretion disk rim being the sole source of the observed IR excess. $^{27}$

Figure 8 shows the comparable relation between orbital period and IR excess at 5.7 and $7.9 \mu \mathrm{m}$. V442 Oph and WX Ari are, again, outliers in the $7.9 \mu \mathrm{m}$ data set (less so in the $5.7 \mu \mathrm{m}$ data set). The linear fits to the complete data sets at both wavelengths, weighted by the uncertainties of both parameters, have negative slopes; however, they are consistent with zero within $\approx 1 \sigma$ (see Table 4 ). After excluding the two outliers, the fit slopes are significantly more shallow (consistent with zero within $\ll 1 \sigma)$, implying that there is not a strong relationship between these two parameters. The Spearman's rank coefficient shows a negative correlation in both cases. The corresponding values of $\rho$ are smaller than for the correlations with inclination, and close to zero when the two outliers are excluded. Additionally, there is a high probability that the correlations at both wavelengths (both with and without the outliers) could have occurred by chance. Thus, there appears to be no statistically significant correlation between orbital period and IR excess. This implies that the strength of the IR excess is not strongly linked to differences in mass transfer rate caused by secular evolution of the CVs (keeping in mind that the NLs generally have high mass transfer rates compared to other

\footnotetext{
27 Not to mention that the standard model predicts temperatures of $\gtrsim 6000 \mathrm{~K}$ for the accretion disk rims of the NLs modeled in this work-consistent with disk rim temperatures found by other modeling work (Linnell et al. 2007a, 2008a, 2008b, 2010, 2012)—whereas the IR excess corresponds to much cooler thermal emission ( $\lesssim 1500 \mathrm{~K})$. Cooler disk rim temperatures cannot be achieved within the constraint that the accretion disk must have a maximum radius no larger than the WD's Roche lobe (and, in fact, somewhat smaller still due to tidal truncation of the disk-see the review in Section 2.5.5 of Warner 2003 and references therein).
}

CVs, so a link between orbital period and/or mass transfer rate and the IR excess could be a higher order effect that is not apparent here).

\subsection{Spectral Energy Distribution Models}

In this section, we present physically realistic, multicomponent models calculated as in Hoard et al. (2009) to reproduce the IR SEDs of several representative members of our target sample. The similarity of the SEDs (see Figure 6), parameter degeneracies of the accretion disk and circumbinary dust disk model components (see below), and the fact that almost all of these targets have well-determined physical parameters or complete system models already in the literature, suggests that there would be a limited scientific return on reproducing the SEDs of all of the CVs in our sample. Rather, we can infer from a few representative models that targets with similar SEDs will have similar models and similar components that fit their IR excess. Parameters used for the individual models discussed below are listed in Table 5; published system parameters for the other targets are listed in Table 6 . The following information applies to all of the models.

1. The WD is represented by a blackbody curve of the desired temperature $\left(T_{\mathrm{wd}}\right)$, normalized to the radius $\left(R_{\mathrm{wd}}\right)$ implied by the mass of the WD $\left(M_{\mathrm{wd}}\right)$.

2. The secondary star is the semi-empirical template with spectral type $(\mathrm{Sp}[2])$, temperature $\left(T_{2}\right)$, and mass $\left(M_{2}\right)$ appropriate for the target's orbital period from Knigge et al. (2011), who performed a comprehensive analysis of the physical properties of the secondary stars across the entire population of $\mathrm{CV}$ s. The templates provided in Knigge et al. (2011) include only the $L(3.5 \mu \mathrm{m}), L^{\prime}(3.8 \mu \mathrm{m})$, and $M(4.80 \mu \mathrm{m})$ bands in the IR. Consequently, we have calculated appropriate synthetic photometry for the WISE and Spitzer (IRAC, MIPS-24) bands to substitute for the $L$, $L^{\prime}, M$ band values in the published templates. These new photometric values were calculated as described in Knigge (2006) and Knigge et al. (2011), but using the BT-Settl model atmospheres ${ }^{28}$ (Allard et al. 2003, 2007, 2012) with

\footnotetext{
28 See http://phoenix.ens-lyon.fr/simulator/index.faces.
} 
Table 5

Model Parameters for Selected Targets

\begin{tabular}{|c|c|c|c|c|}
\hline Star & V592 Cas & IX Vel & UX UMa & RW Sex \\
\hline$T_{\mathrm{wd}}(\mathrm{kK})$ & $45[1,2]$ & $60(10)[1,3]$ & $20[1,4]$ & $50[1,5]$ \\
\hline$M_{\mathrm{wd}}\left(M_{\odot}\right)$ & $0.75[1,2]$ & $0.8(2)[1,3]$ & $0.47[1,4,6],(0.83-1.01) \pm 0.20[7]$ & $0.90[1,5]$ \\
\hline$R_{\mathrm{wd}}\left(R_{\odot}\right)$ & $0.0106[1,2]$ & $0.0150[1,3]$ & $0.0165[1,4], 0.0139[6]$ & $0.00882[1,5]$ \\
\hline $\mathrm{Sp}[2]$ & M4.1 $[1,8]$ & M2.6 [1,8] & M2.6 $[1,8]$ & $\approx \mathrm{M} 1[1,8]$ \\
\hline$T_{2}(\mathrm{~K})$ & $3293[1,8]$ & $3500(1000)[3], 3614[1,8]$ & $3623[1,8]$ & $\gtrsim 3908[1,8]$ \\
\hline$M_{2}\left(M_{\odot}\right)$ & $0.200[1,8]$ & $0.52(10)[3], 0.448[1,8]$ & $0.47[4,6],(0.36-0.43) \pm 0.10[7], 0.459[1,8]$ & $\gtrsim 0.599[1,8], 0.674[5]$ \\
\hline$\dot{M}\left(10^{-9} M_{\odot} \mathrm{yr}^{-1}\right)$ & $15[1,2]$ & $5.0 \pm 1.0[3], 7.1[1,8]$ & $5-10[4], 6-16[6], 8.0[1]$ & $2.0[1,5], 5.75[5]$ \\
\hline$R_{\text {acd,in }}\left(R_{\mathrm{wd}}\right)$ & $1.00[1,2]$ & $1.00[1,3]$ & $1.00[1,4]$ & $1.00[1,5]$ \\
\hline$R_{\text {acd,out }}\left(R_{\mathrm{wd}}\right)$ & $35.00[1,2]$ & $37.33[1,3]$ & $29.58[1,4], 29.90[6]$ & $40.00[1], 50.00[5]$ \\
\hline$h_{\mathrm{acd}, \text { in }}\left(R_{\mathrm{wd}}\right)$ & $0.05[1,2]$ & $0.178[1]$ & $0.039[1]$ & $0.05[1]$ \\
\hline$h_{\text {acd,out }}\left(R_{\mathrm{wd}}\right)$ & $1.68[1,2]$ & $13.33[1,3]$ & $0.96[1,4]$ & $4.2[1]$ \\
\hline$f_{\mathrm{acd}, 3.55}(\%)$ & 93 & 78 & 54 & 50 \\
\hline$f_{\text {acd }, 7.87}(\%)$ & 77 & 69 & 42 & 38 \\
\hline$f_{\text {acd,23.68 }}(\%)$ & 26 & 53 & 26 & 29 \\
\hline$T_{\text {cbd,in }}(\mathrm{K})$ & $500[1,2]$ & $1000[1]$ & $1000[1]$ & $800[1]$ \\
\hline$T_{\text {cbd,out }}(\mathrm{K})$ & $50[1,2]$ & $20[1]$ & $20[1]$ & $20[1]$ \\
\hline$R_{\mathrm{cbd}, \text { in }}\left(R_{\mathrm{wd}}\right)$ & $700[1,2]$ & $172[1]$ & $143[1]$ & $352[1]$ \\
\hline$R_{\text {cbd,out }}\left(R_{\mathrm{wd}}\right)$ & $15000[1,2]$ & $31677[1]$ & $26344[1]$ & $48110[1]$ \\
\hline$M_{\text {cbd,total }}\left(10^{21} \mathrm{~g}\right)^{\mathrm{a}}$ & $2.30[1,2]$ & $1.29[1]$ & $1.40[1]$ & $1.1[1]$ \\
\hline$f_{\mathrm{cbd}, 3.55}(\%)$ & $<1$ & 1 & 3 & $<1$ \\
\hline$f_{\text {cbd }, 7.87}(\%)$ & 15 & 10 & 18 & 7 \\
\hline$f_{\text {cbd, } 23.68}(\%)$ & 72 & 33 & 51 & 32 \\
\hline
\end{tabular}

Notes. The $f_{\text {acd }, \lambda}$ and $f_{\text {cbd, } \lambda}$ parameters are the fraction of total modeled system light contributed by the accretion disk and circumbinary disk components, respectively, at the indicated wavelength (corresponding to IRAC-1, IRAC-4, or MIPS-24).

a Total dust mass corresponds to grains with radius of $r_{\text {grain }}=1 \mu \mathrm{m}$ and scales with $r_{\text {grain }}$.

References. Listed in brackets with each parameter value: $1=$ this work; $2=$ Hoard et al. (2009); $3=$ Linnell et al. (2007a); $4=$ Linnell et al. (2008a); $5=$ Linnell et al. (2010) — the smaller (larger) $\dot{M}$ corresponds to the smaller (larger) distance estimate listed in Table $1 ; 6=$ Noebauer et al. $(2010) ; 7=$ Neustroev et al. (2011) — the larger component mass estimates correspond to the lower inclination from Table 1; 8= Knigge et al. (2011).

Table 6

Published System Parameters

\begin{tabular}{|c|c|c|c|c|}
\hline Star & QU Car & TT Ari & RW Tri & V347 Pup \\
\hline$T_{\mathrm{wd}}(\mathrm{kK})$ & $55[1]$ & $\cdots$ & $\cdots$ & $\cdots$ \\
\hline$M_{\mathrm{wd}}\left(M_{\odot}\right)$ & $0.60,1.20[1]$ & $1.24(20)[2]$ & $0.4-0.7[3], 0.7(1)[4,5], 0.55[6]$ & $0.63(4)[7]$ \\
\hline$R_{\mathrm{wd}}\left(R_{\odot}\right)$ & $0.0159,0.00666[1]$ & $\ldots$ & $0.0115[5]$ & $0.0112(1)[8]$ \\
\hline $\mathrm{Sp}[2]$ & $<\mathrm{M} 1[9]$ & M4.1 [9] & M1.5 [9] & M0.5V [7], K0-5V [8], M1.5[9] \\
\hline$T_{2}(\mathrm{~K})$ & $3500,6500[1],>3900[9]$ & $3311[9]$ & $3892[9]$ & $3894[9]$ \\
\hline$M_{2}\left(M_{\odot}\right)$ & $0.50,1.2[1],>0.6[9]$ & $0.23(2)[2], 0.208[9]$ & $0.3-0.4[3], 0.6[4,5], 0.35[6], 0.594$ [9] & $0.52(6)[7], 0.594[9]$ \\
\hline$\dot{M}\left(10^{-9} M_{\odot} \mathrm{yr}^{-1}\right)$ & $100-1000[1]$ & $1[10]$ & $10[4], 2.7-26,8$ (preferred) [5], $4.6[6]$ & $\sim 6[11], \approx 10[12]$ \\
\hline$R_{\text {acd,in }}\left(R_{\mathrm{wd}}\right)$ & $1.00,1.00[1]$ & $\cdots$ & $1.00[5]$ & $\cdots$ \\
\hline$R_{\text {acd,out }}\left(R_{\mathrm{wd}}\right)$ & $52.83,149.85[1]$ & $\cdots$ & $30.00[5]$ & $0.72(9)[7]$ \\
\hline$h_{\mathrm{acd}, \text { in }}\left(R_{\mathrm{wd}}\right)$ & $\ldots$ & $\cdots$ & $\cdots$ & $\cdots$ \\
\hline$h_{\text {acd,out }}\left(R_{\mathrm{wd}}\right)$ & $3.14,7.51[1]$ & $\cdots$ & $\cdots$ & $\cdots$ \\
\hline Star & V3885 Sgr & VY Scl & V442 Oph & WX Ari \\
\hline$T_{\mathrm{wd}}(\mathrm{kK})$ & $57(5)[13]$ & $45(3)[14]$ & $23[12]$ & $\cdots$ \\
\hline$M_{\mathrm{wd}}\left(M_{\odot}\right)$ & $0.7(1)[13]$ & $1.0[14], 1.22(22)[15]$ & $0.4[12]$ & $\approx 0.8[16], 0.35[12]$ \\
\hline$R_{\mathrm{Wd}}\left(R_{\odot}\right)$ & $0.0134[13]$ & $\cdots$ & $\ldots$ & $\ldots$ \\
\hline $\mathrm{Sp}[2]$ & $\mathrm{M} 2.2[9]$ & M1.5 [9] & M4.0 [9] & M4.1 [9] \\
\hline$T_{2}(\mathrm{~K})$ & $3650(100)[13], 3698$ [9] & $3896[9]$ & 3289 [9] & $3319[9]$ \\
\hline$M_{2}\left(M_{\odot}\right)$ & 0.475 [13], 0.504 [9] & $0.43(13)[15], 0.595$ [9] & $0.200[9]$ & $\approx 0.31[16], 0.306[9]$ \\
\hline$\dot{M}\left(10^{-9} M_{\odot} \mathrm{yr}^{-1}\right)$ & $5(2)[13]$ & $8[14]$ & $10[12]$ & $1[12,16]$ \\
\hline$R_{\mathrm{acd}, \text { in }}\left(R_{\mathrm{wd}}\right)$ & $1.00[13]$ & $1.05[14]$ & $\cdots$ & $\cdots$ \\
\hline$R_{\text {acd,out }}\left(R_{\mathrm{wd}}\right)$ & $41.79[13]$ & $\cdots$ & $\cdots$ & $\cdots$ \\
\hline$h_{\mathrm{acd}, \text { in }}\left(R_{\mathrm{wd}}\right)$ & $\ldots$ & $\cdots$ & $\cdots$ & $\cdots$ \\
\hline$h_{\text {acd,out }}\left(R_{\mathrm{wd}}\right)$ & $7.46[13]$ & $\cdots$ & $\cdots$ & $\cdots$ \\
\hline
\end{tabular}

References. Listed in brackets with each parameter value: $1=$ Linnell et al. (2008b) — the higher mass WD model is preferred, but actual system parameters could lie inside the range of parameter values; $2=$ Wu et al. (2002); $3=$ Poole et al. (2003); $4=$ Groot et al. (2004); $5=$ Noebauer et al. (2010); $6=$ Puebla et al. (2011); 7 = Thoroughgood et al. (2005); $8=$ Diaz \& Hubeny (1999); $9=$ Knigge et al. (2011); 10= Ballouz \& Sion (2009); 11 = Puebla et al. (2007); 12 = Ballouz \& Sion (2009); 13 = Linnell et al. (2009); $14=$ Hamilton \& Sion (2008); $15=$ Martínez-Pais et al. (2000); 16 = Rodríguez-Gil et al. (2000). 
the grid of secondary star physical parameters from Table 6 in Knigge et al. (2011).

3. The accretion disk (ACD) component assumes an optically thick steady state disk composed of concentric rings emitting as blackbodies, following the standard model prescription in Frank et al. (2002). The accretion disk component is parameterized by inner and outer radii ( $R_{\text {acd,in }}$ and $\left.R_{\text {acd,out }}\right)$, vertical semi-height at the inner radius $\left(h_{\text {acd }}\right)$, and a mass transfer rate from the secondary star $(\dot{M})$. The accretion disk is composed of 100 concentric rings, each of which contains 360 azimuthal sections.

The temperature of each disk ring is determined from the standard model radial temperature profile $\left(T \propto r^{-\gamma}\right.$ where $r$ is radial distance from the disk center and $\gamma=0.75$; Frank et al. 2002). The disk is flared such that the height of the disk for radii larger than $R_{\text {crit }}=\left[\left(7-8 \gamma_{\text {out }}\right) /\left(6-8 \gamma_{\text {out }}\right)\right]^{2} R_{\mathrm{wd}}$ increases as $r^{9 / 8}$ (Warner 2003). In this case, $\gamma_{\text {out }}<0.75$ provides a shallower temperature gradient in the outer disk corresponding to irradiation of the inner face of the flared disk by the inner disk and WD (Orosz \& Wade 2003); we use $\gamma_{\text {out }}=0.70$ (Hoard et al. 2009). The vertical outer edge of the disk, which does not "see" the WD and inner accretion disk, is assumed to have a non-irradiated temperature appropriate for the standard model radial temperature profile. Depending on the system inclination and disk geometry, the model accounts for self-occultation of the inner disk by excluding the flux contribution of regions that are blocked from view by the flared disk.

A wavelength- and temperature-dependent correction for limb darkening of the accretion disk is applied to each azimuthal section using linear limb-darkening coefficients interpolated over the grid provided by van Hamme (1993). In the case of a flat disk, this would be a relatively simple procedure, as the disk face everywhere presents the same viewing angle (i.e., angle between the line of sight and a normal to the disk face), so only the disk edge is limb-darkened across a range of viewing angles. However, in the case of the flared disk used here, which presents different viewing angles for different parts of the disk, limb darkening is calculated individually for each azimuthal section in each ring. The primary effect of limb darkening is to make the short wavelength (UV-optical) end of the SED fainter relative to the long wavelength (IR) end.

As found by Orosz \& Wade (2003) and other studies of irradiation in model accretion disk SEDs (e.g., Wade 1988), we found that irradiation (like limb darkening) primarily affects the short wavelength end of the SED. However, irradiation (which effectively raises the temperature at a given radius over the non-irradiated case) tends to make the short wavelength end of the SED brighter relative to the long wavelength end, which somewhat counteracts the effect of limb darkening.

4. The circumbinary dust disk (CBD) component is calculated as described in Hoard et al. (2007, 2009), under the assumption that the disk is optically thin and composed of spherical grains with characteristic radius of $r_{\text {grain }}=1$ $\mu \mathrm{m}$ and density of $\rho_{\text {grain }}=3 \mathrm{~g} \mathrm{~cm}^{-3}$ that (re)radiate as blackbodies. The inner edge of the disk is fixed at the tidal truncation limit ( $R_{\mathrm{cbd}, \text { in }} \approx 1.5$ times the binary separation), and the outer edge $\left(R_{\mathrm{cbd} \text {,out }}\right)$ is fixed at the distance at which the temperature of the dust merges with the ambient interstellar medium at a temperature of $20 \mathrm{~K}$. The input parameters for the CBD component are only the inner edge temperature, $T_{\text {cbd,in }}$ (keeping in mind that silicate dust will sublimate at temperatures higher than $\approx 1500-2000 \mathrm{~K}$; e.g., Pollack et al. 1994; Kobayashi et al. 2011) and the exponent of the radial temperature profile $(\gamma=0.75)$.

As noted in Hoard et al. (2007, 2009), the degeneracies among parameters in the CBD model mean that any given model is representative of a class of similar possible solutions, but is not necessarily a unique solution. Of particular note in the context of the work presented here is the degeneracy between $r_{\text {grain }}$ and total dust mass $\left(M_{\text {cbd,total }}\right)$ for constant $\rho_{\text {grain }}$. The total dust mass values for the models listed in Table 5 were calculated assuming a characteristic dust grain radius of $1 \mu \mathrm{m}$. For constant grain mass density, the total dust mass required to produce an identical CBD SED component scales with $r_{\text {grain }}$.

The use of a characteristic grain radius is a simplifying assumption that can be interpreted as a representative (or approximate mean) value among a distribution of sizes, for those grains that predominantly contribute to the observed SED (e.g., see discussion in the Appendix of Jura et al. 2009). On a grain-by-grain basis, the emitted spectrum of a dust grain in temperature equilibrium is a blackbody modified by a function that depends on wavelength (as well as temperature, grain size and composition; e.g., Dwek et al. 1980; Draine 1981). This function is approximately a constant (i.e., independent of wavelength) for $\lambda \lesssim \pi r_{\text {grain }}$ (i.e., $\sim 3-30 \mu \mathrm{m}$ for $1-10 \mu \mathrm{m}$ grains), and varies as $\lambda^{-1}-\lambda^{-2}$ at wavelengths much larger than the grain size, $\lambda \gtrsim 5 \pi r_{\text {grain }}$ (i.e., $\sim 16-160 \mu \mathrm{m}$ for $1-10 \mu \mathrm{m}$ grains; e.g., Carciofi et al. 2004). Nonetheless, in the case of dust disks around single WDs, blackbody SED models utilizing an appropriate radial temperature profile adequately reproduce the broadband IR photometric observations (e.g., Jura 2003; Farihi 2011), under the assumption that the grains are predominantly up to $\sim$ a few $\mu \mathrm{m}$ in size (based on the presence of the $10 \mu \mathrm{m}$ silicate emission feature-see additional discussion of this in Section 4.1.1) with a characteristic size of $\sim 1 \mu \mathrm{m}$ (Jura et al. 2009). If the distribution of sizes is dominated by grains that are much smaller $(\lesssim 0.1 \mu \mathrm{m})$ or much larger ( $\gtrsim 100 \mu \mathrm{m})$, then the resultant near- to mid-IR spectrum could deviate significantly from the expectation of simple blackbody radiation.

5. For a given target, all model components are normalized to the estimated distance to the target $(d)$ and are assumed to share a single inclination $(i)$. The values of $d$ and $i$ are taken from best estimates in the literature (see Table 1) and, in the interests of improving the degree to which the available data can constrain the models, are not allowed to vary freely.

The $f_{\text {acd }, \lambda}$ and $f_{\text {cbd, } \lambda}$ values listed in Table 5 are the fractions of the total modeled system light contributed by the accretion disk and circumbinary disk components, respectively, at the indicated wavelengths. The contribution from the WD is negligible at the tabulated IR wavelengths, so the secondary star contribution can be inferred by subtracting the sum of the tabulated fractions from $100 \%$.

\subsubsection{Model for V592 Cas}

Figure 9 shows our model for V592 Cas from Hoard et al. (2009) overlaid on the re-extracted Spitzer photometry discussed here (along with 2MASS and WISE). The only difference from the Hoard et al. (2009) treatment is that we have used the updated 


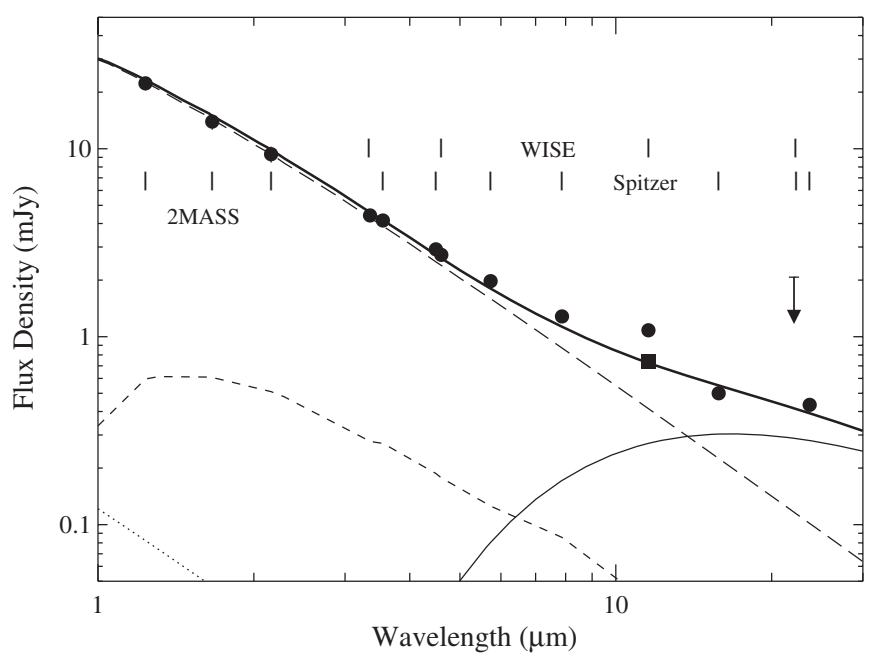

Figure 9. IR SED of V592 Cas using the 2MASS (dereddened), WISE, and reextracted Spitzer photometry (circles), along with the multi-component system model from Hoard et al. (2009) and this work. The total system model (thick solid line) is composed of a WD (dotted line), M4.1 secondary star (short dashed line; semi-empirical template from Knigge et al. 2011 with additional IR points), limb-darkened, flared and irradiated, steady state accretion disk (long dashed line), and circumbinary dust disk (thin solid line). The isophotal wavelengths of the 2MASS, WISE, and Spitzer (IRAC, IRSPUI, MIPS-24) bands are indicated. The WISE W4 point is a non-detection upper limit. The single square point is our PSF-fit photometry for the WISE W3 band (see Section 2.2.1).

secondary star model from Knigge et al. (2011) appropriate for the orbital period of V592 Cas (M4.1; see Table 5) this requires a slightly larger (by $\approx 4 \%$ ) distance than used in Hoard et al. (2009) to scale the model to the data. There is no significant disagreement with the model due to the slightly different photometry yielded by the final Spitzer cryogenic flux calibration. Unfortunately, the Herschel upper limits at 70 and $160 \mu \mathrm{m}$ for V592 Cas, $\leqslant 1.1 \mathrm{mJy}$ and $\leqslant 4.5 \mathrm{mJy}$, respectively (Harrison et al. 2013), are not helpful in constraining the presence and characteristics of dust. They are more than an order of magnitude brighter than the extrapolation of the V592 Cas SED model, which gives a total predicted system flux density at $\gtrsim 70 \mu \mathrm{m}$ of $<0.1 \mathrm{mJy}$ (dominated by the CBD component).

\subsubsection{Model for IX Vel}

Linnell et al. (2007a) calculated a model fit to the UV-optical SED of IX Vel with the BINSYN multi-component model atmosphere/synthetic spectrum code (Linnell \& Hubeny 1996; Linnell et al. 2008b, 2012). We utilize their system parameters as starting points (see Table 5) to construct our IR SED model. Figure 10 shows our model for IX Vel, composed of WD, secondary star, accretion disk, and CBD components.

In Section 2.2.2, we applied a correction to the Cycle-5 IRS spectrum of IX Vel to bring it into agreement with the brighter Cycle-2 IRS spectrum. We now demonstrate that the assumed spectral shape of this correction (i.e., a RJL $f_{v} \propto \lambda^{-2}$ ), which corresponds to the assumption that the overall brightness change is dominated by a change in the accretion disk modulated by $\dot{M}$, is justified. Figure 11 shows a close-up of the IR region of the IX Vel SED and model shown in Figure 10. The Cycle-2 Spitzer data are plotted in black, and the uncorrected (fainter) Cycle-5 IRS spectrum is plotted in gray. The secondary star (short dashed line) and CBD (thin solid line) components are unchanged from the model shown in Figure 10 (and described above), as are the brighter accretion disk (upper long dashed line) and system (upper thick solid line) models. The fainter

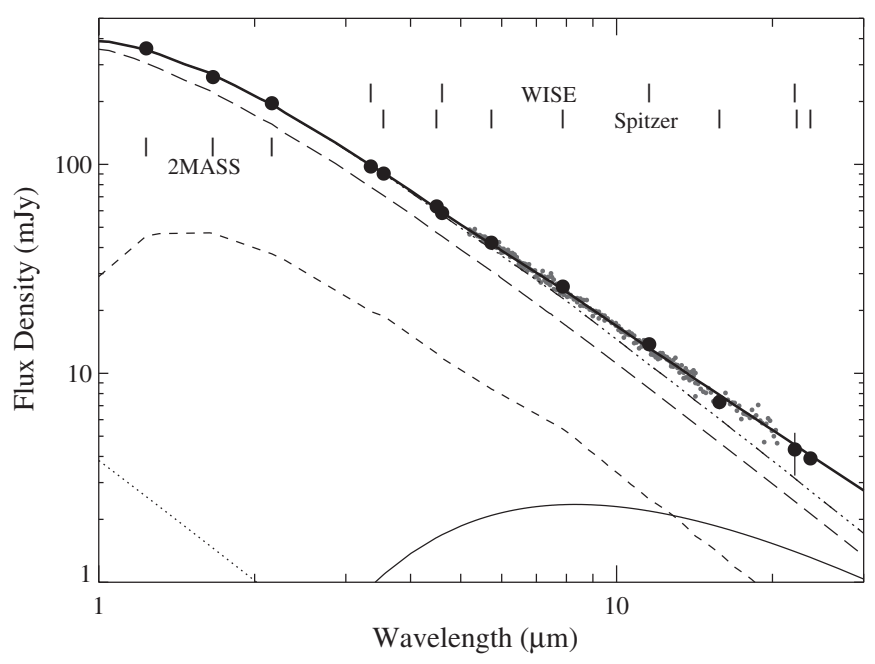

Figure 10. IR SED of IX Vel using the 2MASS, WISE, Spitzer photometry (circles), and Spitzer spectrum (small circles), along with our multi-component system model. The total system model (thick solid line) is composed of a WD (dotted line), M2.6 secondary star (short dashed line; semi-empirical template from Knigge et al. 2011 with additional IR points), limb-darkened, flared and irradiated, steady state accretion disk (long dashed line), and circumbinary dust disk (thin solid line). The system model utilizes the parameters of Linnell et al. (2007a; see Tables 1 and 5). The dot-dot-dot-dash line is not an additional model component; instead, it shows the model without the contribution of the circumbinary dust disk. The isophotal wavelengths of the 2MASS, WISE, and Spitzer (IRAC, IRSPUI, MIPS-24) bands are indicated.

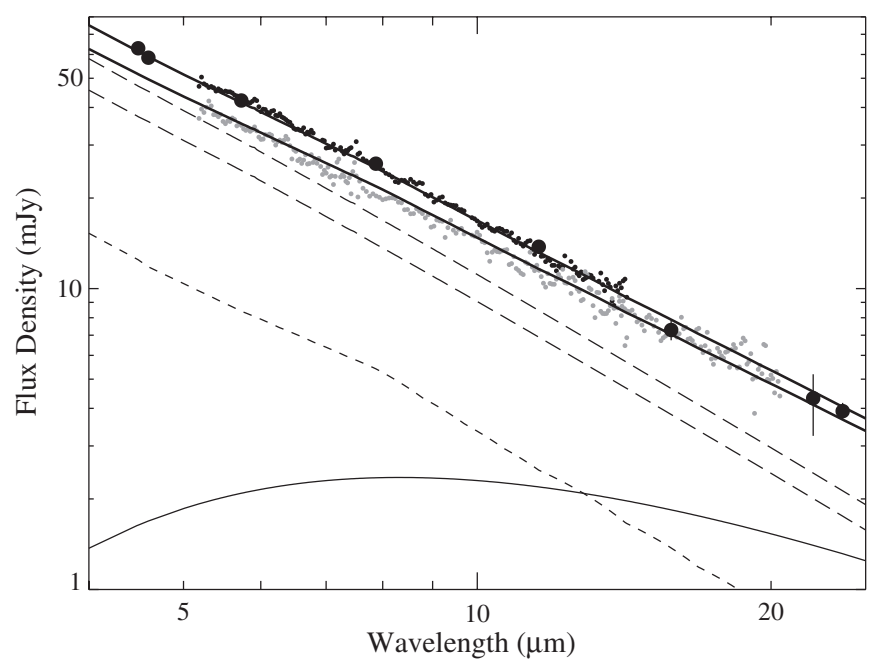

Figure 11. Comparison of accretion disk models for IX Vel. This is a close-up of the IR region of the model shown in Figure 10, with the Cycle-2 Spitzer data (black points) and corresponding secondary star (short dashed line), CBD (thin solid line), accretion disk (upper long dashed line), and total system (upper thick solid line) model SEDs. The uncorrected Cycle-5 IRS spectrum (small gray points) is plotted along with a fainter accretion disk model component (lower long dashed line) that yields a total system model (lower thick solid line) in agreement with the Cycle-5 spectrum. See Section 3.3.2 for additional discussion.

accretion disk model component (lower long dashed line) has $\dot{M}=3.4 \times 10^{-9} M_{\odot} \mathrm{yr}^{-1}$, which is $\approx 50 \%$ of the bright accretion disk value. The corresponding faint system model (lower thick solid line) agrees well with the uncorrected Cycle-5 data.

The difference between the two accretion disk components differs from a pure $\lambda^{-2}$ dependence by less than $4 \%$ at all wavelengths in the range 5-20 $\mu \mathrm{m}$. Hence, the functional form of the correction is reasonable in the context of the assumption stated above. In any case, the outcome of applying the RJL 


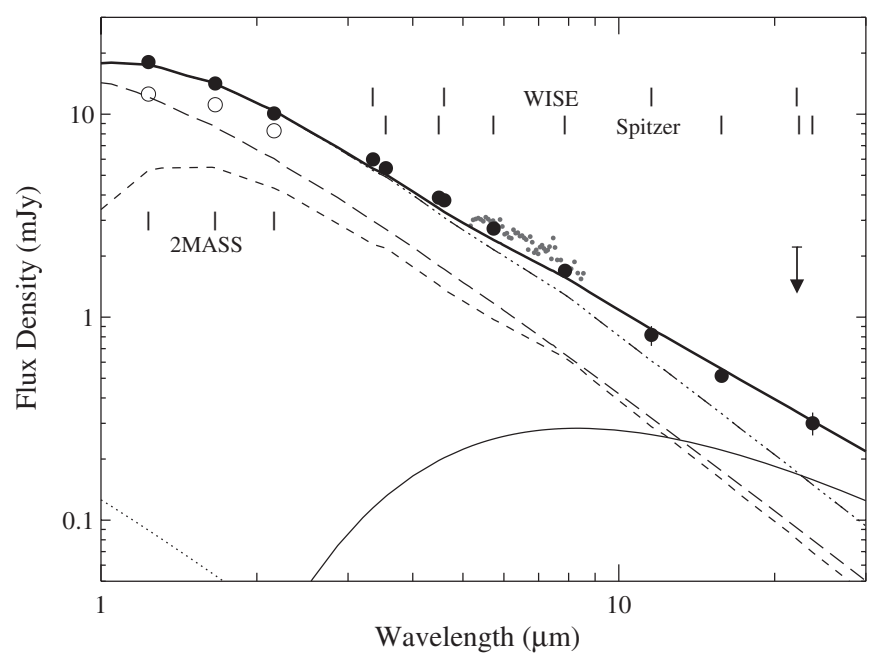

Figure 12. IR SED of UX UMa using the 2MASS, WISE, Spitzer photometry (circles), and Spitzer spectrum (small circles), along with our multi-component system model. The total system model (thick solid line) is composed of a WD (dotted line), M2.6 secondary star (short dashed line; semi-empirical template from Knigge et al. 2011 with additional IR points), limb-darkened, flared and irradiated, steady state accretion disk (long dashed line), and circumbinary dust disk (thin solid line)—see model parameters in Table 5. An offset has been applied to the 2MASS points, as described in Section 3.3.3; the original 2MASS photometry is shown as unfilled circles. The dot-dot-dot-dash line is not an additional model component; instead, it shows the model without the contribution of the circumbinary dust disk. The isophotal wavelengths of the 2MASS, WISE, and Spitzer (IRAC, IRSPUI, MIPS-24) bands are indicated.

correction here is primarily cosmetic-none of the IR excess detections or modeling results depend solely on data that were corrected in this manner. Incidentally, we note that a decrease in the accretion rate by a factor of two corresponds to a decrease in the total disk luminosity by a factor of two (Frank et al. 2002); comparing the two accretion disk models for IX Vel on a wavelength-by-wavelength basis shows that the corresponding change in the total system brightness would be $\Delta R \approx 0.5 \mathrm{mag}$ and $\Delta K \approx 0.3 \mathrm{mag}$.

\subsubsection{Model for UX UMa}

Linnell et al. (2008a) calculated a model fit to the UV-optical SED of UX UMa, using the BINSYN code as for IX Vel. However, in the case of UX UMa, they were not able to completely resolve a degeneracy between mass transfer rate and distance, finding a range of equally acceptable solutions for $d=250-345 \mathrm{pc}$ and $\dot{M}=5-10 \times 10^{-9} M_{\odot} \mathrm{yr}^{-1}$. We started our modeling process using their suggested compromise values of $d=312 \mathrm{pc}$ and $\dot{M}=8 \times 10^{-9} M_{\odot} \mathrm{yr}^{-1}$, as well as the other system parameters derived in their study (see Table 5). However, it quickly became apparent that there was a flux density offset between the 2MASS and Spitzer+WISE data, with the former being fainter than expected compared to the latter. The AAVSO long-term light curve of UX UMa shows that there was no significant difference in mean brightness during the 2MASS observations compared to the later Spitzer and WISE observations. However, UX UMa-like other CVs-does display stochastic brightness variations, on timescales of days, with an amplitude of $0.2-0.3 \mathrm{mag}$ (in V). When we utilize only the Spitzer and WISE data, we obtain a good model for the IR SED using values of $d=290 \mathrm{pc}$ and $\dot{M}=7 \times 10^{-9} M_{\odot} \mathrm{yr}^{-1}$, consistent with the range found by Linnell et al. (2008a); however, the degeneracy between distance and $\dot{M}$ persists so this should be regarded as a representative, rather than unique,

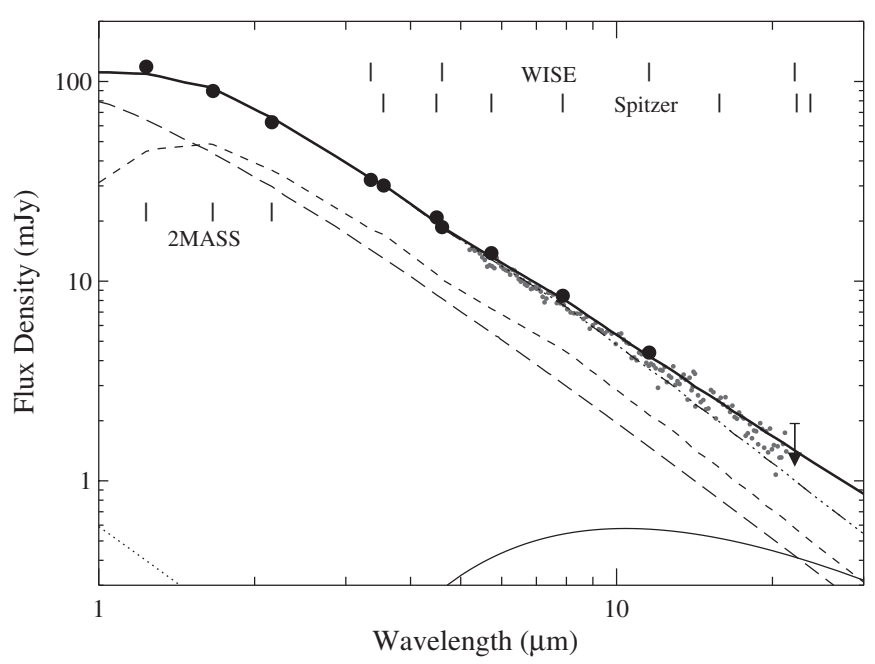

Figure 13. IR SED of RW Sex using the 2MASS, WISE, Spitzer photometry (circles), and Spitzer spectrum (small circles), along with our multi-component system model. The total system model (thick solid line) is composed of a WD (dotted line), M1 secondary star (short dashed line; semi-empirical template from Knigge et al. 2011 with additional IR points), limb-darkened, flared and irradiated, steady state accretion disk (long dashed line), and circumbinary dust disk (thin solid line) - see model parameters in Table 5. The dot-dot-dot-dash line is not an additional model component; instead, it shows the model without the contribution of the circumbinary dust disk. The isophotal wavelengths of the 2MASS, WISE, and Spitzer (IRAC, IRSPUI, MIPS-24) bands are indicated.

solution. Adding a RJL offset to the 2MASS photometry, with an initial value of $+5.5 \mathrm{mJy}$ at the $J$ band, brings it into conformity with the model. We note that, in this case, the appropriateness of applying this correction is less certain, since the peak of the secondary star component makes a non-negligible $(\approx 30 \%)$ and non-RJL contribution to the total system brightness in the 2MASS bands. We plot both the original and corrected 2MASS data in Figure 12, which shows our SED model.

\subsubsection{Model for RW Sex}

We started our modeling process for RW Sex using the favored BINSYN model parameters from Linnell et al. (2010) (see Table 5). The orbital period of this NL (353 min) is slightly longer than the longest orbital period for which Knigge et al. (2011) provide a semi-empirical secondary star template (336 minutes). To compensate for this, we used the longest period secondary star template from Knigge et al. (2011), scaled up in brightness by $15 \%$, which is the approximate difference between the longest period secondary star template and the secondary star template corresponding to an orbital period that is shorter by the same amount as the orbital period of RW Sex is longer. The resultant secondary star model component is slightly brighter than the accretion disk component obtained using the size and mass transfer rate parameters from Linnell et al. (2010). Figure 13 shows the complete SED model for RW Sex, utilizing the scaled Knigge et al. (2011) secondary star template and a CBD component to model the IR excess apparent at wavelengths longer than $\approx 8 \mu \mathrm{m}$.

\section{DISCUSSION}

The IR SEDs of the eleven NLs presented here are largely similar to that of V592 Cas and all show an IR excess. This suggests that the IR excess in all cases could have a similar origin. We have demonstrated here (and in Hoard et al. 2009) that physically realistic, multi-component models utilizing emission 
from circumbinary dust disks can reproduce the observed SEDs. At the same time, several of the targets observed here are believed to have significant accretion disk wind outflows, which can even exceed the WD escape velocity (e.g., see Howell et al. 1995; Prinja \& Rosen 1995, and Appendices A.1 and A.4), and could provide a suitable environment for the generation of bremsstrahlung (free-free emission) that also might produce an IR excess. Harrison et al. (2013) investigated the mid- to far-IR properties of a sample of primarily DNe and magnetic CVs, with only the NL V592 Cas in common with our study. They suggest that bremsstrahlung contributes significantly to the observed IR excesses in V592 Cas and the archetype DN SS Cygni, with a possible additional contribution from dust only in V592 Cas. Thus, we should devote some consideration to the relative merits of emission from circumbinary dust versus bremsstrahlung as the explanation for the IR excesses in these objects.

It should first be noted, however, that there is a potentially serious drawback to the modeling approach used by Harrison et al. (2013). To reproduce the optical-IR SEDs of their sample of CVs (including V592 Cas), Harrison et al. (2013) utilized a self-described "simple" model intended to avoid the difficulty in constraining a multi-component model (as in Hoard et al. 2009 and this work). They simply utilized two blackbodies, one representing all of the hot, optically thick regions in the $\mathrm{CV}$ (i.e., WD, boundary layer, accretion disk, etc.), and a second blackbody representing the secondary star. Their justification for this modeling approach is that since all of the components are on the Rayleigh-Jeans tail in the IR, they simply sum together as a power law.

The problem arises from the fact that cool (M type) stars deviate from having Rayleigh-Jeans spectra in the mid-IR (IRAC bands and longer wavelengths) due to molecular pollution in their photospheres (Patten et al. 2006). In fact, the observed $K s-\left[\right.$ MIPS-24] color index for cool stars $\left(T_{\text {eff }}=2000-4000 \mathrm{~K}\right)$ is systematically and significantly smaller than that of a blackbody at the corresponding temperature (see Figure 2 in Gautier et al. 2007). Thus, if the star and the blackbody have the same $K s$ magnitude, then the blackbody will be brighter than the star at $24 \mu \mathrm{m}$. This effect is demonstrated in Figure 14, in which we have plotted several of the Knigge et al. (2011) secondary star templates with our added IR points spanning spectral types of M1.5-M5 (corresponding to CV orbital periods of 5.5-1.9 hr, respectively). We have also plotted a corresponding blackbody function with the same temperature as each secondary star template, scaled to match the $H$-band flux density of the corresponding template. The blackbodies overestimate the SEDs of the secondary stars by $30 \%-60 \%$ in the WISE and Spitzer bands. In V592 Cas, the ultimate effect of this is not severe, since the overall SED is dominated by the accretion disk at all wavelengths (e.g., the blackbody is $\approx 30 \%$ brighter than the secondary star template at $10 \mu \mathrm{m}$, but this difference corresponds to $\approx 2 \%$ of the total system brightness at that wavelength). However, in systems for which the secondary star makes a larger relative contribution, using a blackbody to represent the secondary star could yield a false-negative for the presence of an IR excess. For example, the blackbody "secondary stars" for UX UMa and RW Sex are $\approx 25 \%$ and $\approx 40 \%$ brighter than the Knigge et al. (2011) templates in the IRAC-4 $(7.8 \mu \mathrm{m})$ band. Since the secondary star contributes $\approx 40 \%$ and $\approx 50 \%$ of the total flux for UX UMa and RW Sex, respectively, in the IRAC-4 band, using a blackbody to represent the secondary star would overestimate the corresponding flux density by $10 \%-20 \%$. This is comparable to the strength of the IR excess component at the IRAC- 4 band in

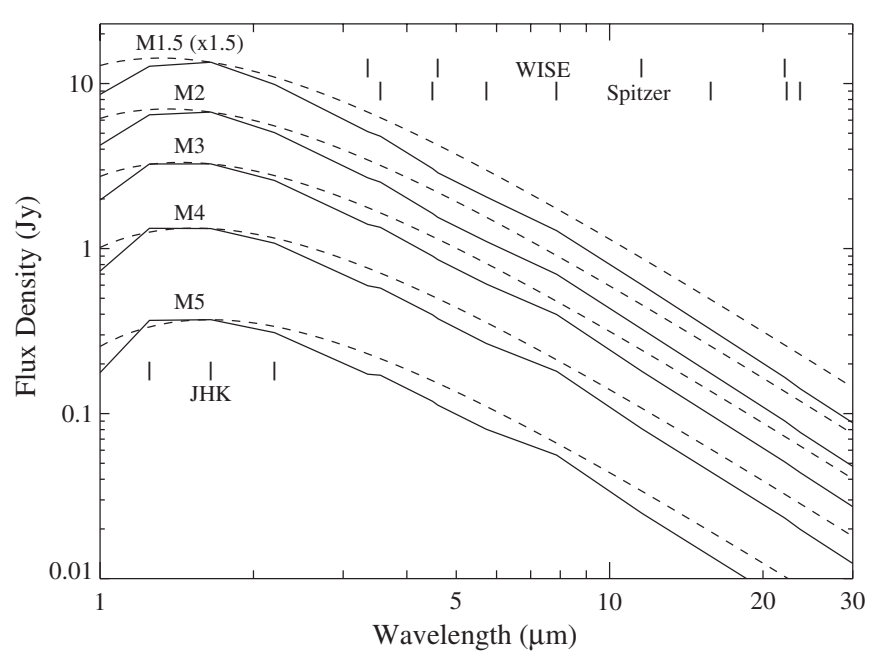

Figure 14. Comparison of the Knigge et al. (2011) semi-empirical secondary star templates with additional IR points used here (solid curves) with blackbodies (dashed curves). The secondary star templates are (top to bottom): M1.5 $\left(P_{\text {orb }}=5.5 \mathrm{hr}, T_{2}=3881 \mathrm{~K}\right), \mathrm{M} 2\left(P_{\text {orb }}=5.2 \mathrm{hr}, T_{2}=3758 \mathrm{~K}\right), \mathrm{M} 3$ $\left(P_{\text {orb }}=4.3 \mathrm{hr}, T_{2}=3532 \mathrm{~K}\right), \mathrm{M} 4\left(P_{\text {orb }}=3.4 \mathrm{hr}, T_{2}=3330 \mathrm{~K}\right)$, and M5 $\left(P_{\text {orb }}=1.9 \mathrm{hr}, T_{2}=3112 \mathrm{~K}\right)$. The flux densities correspond to a distance of $10 \mathrm{pc}$; the SED for spectral type M1.5 was scaled upward by a factor of 1.5 for clarity. The blackbody functions were calculated for the same temperatures as the corresponding secondary star templates, and scaled to match the $H$-band point. The isophotal wavelengths of the $J, H, K, W I S E$, and Spitzer (IRAC, IRSPUI, MIPS-24) bands are indicated.

these two systems (see Table 5), resulting in a false-negative for the presence of an IR excess. The negative results reported for the presence of IR excess in the CVs modeled by Harrison et al. (2013) using blackbody secondary stars (which dominate their model SEDs in the IR) could have been similarly affected and should be treated with caution.

\subsection{Dust or Bremsstrahlung?}

We now compare the principal advantages and disadvantages of the dust and bremsstrahlung mechanisms as explanations for the observed IR excess in CVs. Dust is known to be associated with some phases of CV activity, such as classical nova outbursts (Bode 1982; Evans 1997, 2001; Evans \& Gehrz 2012) and tremendous outburst amplitude DNe (Ciardi et al. 2006). In addition, Bisikalo $(2009,2011)$ and Sytov et al. (2007, 2009) have performed detailed numerical simulations of the mass transfer process in $\mathrm{CVs}$, and find that a significant fraction of the mass transferred from the secondary star into the WD Roche lobe is ejected from the inner binary through the outer Lagrange point $\left(L_{3}\right)$. This material spreads into circumbinary space around the CV (see Figure 5 in Bisikalo 2009), and could provide the building blocks for dust formation (or even already complete dust grains transferred from their formation site in the outer layers of a cool secondary star). Along with other possible delivery mechanisms for material into circumbinary space around the $\mathrm{CV}$, such as the disk and/or secondary star wind and nova and DN outbursts, there appears to be sound justification to expect that dust could form in such an environment.

The bremsstrahlung mechanism can operate in astrophysical environments with conditions similar to those found in regions of a CV (i.e., ionized plasmas). It was previously excluded as the origin of the IR excess in V592 Cas for a number of reasons discussed in Hoard et al. (2009) and references therein (see Section 4.1 in that paper). Notably, the mass loss rate in the 
accretion disk wind required to explain the observed level of IR excess via bremsstrahlung would be $\sim 5 \times 10^{-9} M_{\odot} \mathrm{yr}^{-1}$, more than $30 \%$ of the inferred steady state mass transfer rate in V592 Cas. By comparison, Proga et al. (2002), Noebauer et al. (2010), and Puebla et al. (2011) modeled wind mass loss rates for several high mass transfer rate NLs in common with the targets of our survey: $\lesssim 10^{-10} M_{\odot} \mathrm{yr}^{-1}$ for RW Tri, V347 Pup, and IX Vel, and a higher value of $\sim 1 \times 10^{-9} M_{\odot} \mathrm{yr}^{-1}$ for UX UMa. In all four cases, the wind mass loss rate is $\lesssim 8 \%$ of the mass transfer rate. In fairness, we note that it is currently unclear whether this situation reflects a true generality in the relative values of the mass transfer and disk wind loss rates, or a shortcoming of the disk wind models in reproducing an extremely complex and poorly constrained process.

Nonetheless, we can attempt to ascertain the likely spectral profile shape $\left(f_{v} \propto v^{\alpha} \propto \lambda^{-\alpha}\right)$ of a bremsstrahlung component originating in a $\mathrm{CV}$ wind by comparing theoretical predictions to the observed IR SED of V592 Cas. Using the re-extracted photometry for V592 Cas presented here, the observed value of the flux density ratio for the IR excess component (i.e., exclusive of the WD, secondary star, and accretion disk) between the MIPS-24 $(23.675 \mu \mathrm{m})$ and IRSPUI-blue $(15.8 \mu \mathrm{m})$ bands is $f_{v, 24} / f_{v, 16}=0.65$, corresponding to a spectral index of $\alpha \approx 1.06 .29$ The bremsstrahlung spectral profile is strongly influenced by the characteristics of the emitting plasma and can exhibit a wide range of frequency dependence in different plasma geometries. In the simplest case of an optically thin plasma "slab" at constant density, the wavelength dependence of bremsstrahlung varies in proportion to the Gaunt factor and is approximately flat $(\alpha \approx 0)$. In a spherically symmetric wind outflow at constant velocity (i.e., with density related to radial distance by $\rho \propto r^{-2}$ ), the expected bremsstrahlung spectral index in the IR-radio is $\alpha=0.67-0.6$, respectively (Wright \& Barlow 1975). Barlow \& Cohen (1977) showed that bremsstrahlung originating in an accelerating wind (e.g., near the base of the wind in $\mathrm{O}$ supergiants) should have a spectral index $\alpha>0.67$ in the IR, corresponding to a density distribution in the wind of $\rho \propto r^{-\beta}$, where $\beta>2(\alpha$ and $\beta$ are related by $\alpha=[4 \beta-6] /[2 \beta-1]$; also see Wright $\&$ Barlow 1975). At high optical depth (e.g., in the region where a stellar wind emerges from the photosphere-see Barlow \& Cohen 1977- or possibly in the innermost regions of a CV accretion disk - as suggested by Harrison et al. 2013), bremsstrahlung has a blackbody spectral shape (i.e., $\alpha=2$ on the Rayleigh-Jeans tail in the IR; essentially, it is the observed thermal continuum of the accretion disk).

If the observed excess in the IR SED of V592 Cas is due to bremsstrahlung, then it would be most consistent with the case of an accelerating wind with a density distribution of $\rho \propto r^{-2.6}$. In the case of the other CVs modeled here (see Section 3.3), the flux density ratios of their less prominent IR excess components is $f_{v, 24} / f_{v, 16} \approx 0.70-0.75$, which yields $\alpha \approx 0.9-0.7$, respectively. This value of $\alpha$ is slightly larger than the expectation for bremsstrahlung from a constant velocity wind, and is consistent with the accelerating wind case, at a lower wind density than for V592 Cas. However, this is far from a complete picture of this scenario. The actual bremsstrahlung profile is a function of many additional parameters, including mass flux and terminal velocity of the wind, mean atomic weight of the gas, the Gaunt factor, and so on (see Wright \& Barlow

\footnotetext{
29 In Hoard et al. (2009), the observed value for this ratio was reported as 0.91 , corresponding to $\alpha \approx 0.23$; however, this utilized the older flux calibration of the MIPS and IRSPUI-blue data.
}

1975 and Barlow \& Cohen 1977), and can presumably be a combination of emission from several regions with different conditions. Ultimately, a component that can, in essence, be shaped to fit any observed data is not helpful in discriminating the actual explanation for the observations (but neither does this shortcoming alone rule out bremsstrahlung as a viable mechanism contributing to the IR SED of NLs).

If the IR emission component is due to bremsstrahlung, then we would also expect its properties (especially overall brightness) to be causally tied to the instantaneous accretion disk properties at any given time, primarily the disk brightness (which is, in turn, directly linked to the rate of mass flow through the disk). This is true whether the bremsstrahlung originates in a wind from the disk or in the dense regions of the inner disk itself (as proposed by Harrison et al. 2013). IR emission from circumbinary dust, on the other hand, should be largely independent of stochastic fluctuations in the brightness of the accretion disk around a long-term mean. In that regard, the behavior of IX Vel suggests that the IR excess component is more consistent with emission from circumbinary dust: even when IR spectra observed at different epochs indicated a significant $(\sim 50 \%)$ change in the mass transfer rate through the accretion disk, the additional IR emission component required to reproduce the corresponding SEDs of IX Vel remained unchanged (see Section 3.3.2). This appears to be a significant point in favor of the dust scenario.

\subsubsection{The Missing Emission Feature}

Our five targets with Spitzer IRS spectra that extend past $10 \mu \mathrm{m}$ show no evidence for the broad silicate emission feature that is the hallmark of circumstellar dust around isolated WDs (e.g., Jura et al. 2009). The additional five targets that do not have an IRS spectrum but do have a WISE W3 $(11 \mu \mathrm{m})$ photometric measurement also do not show evidence for enhanced emission in this wavelength region. We have shown in Section 3.1 that the elevated W3 value for V592 Cas in the WISE All Sky Catalog is, in fact, due to contamination from a nearby star. Also as noted above, V442 Oph and WX Ari lack data at $\lambda>8 \mu \mathrm{m}$, so the presence of silicate emission in these two systems that each have a significant IR excess currently cannot be investigated.

This situation might be inductively construed as lending additional credence to the bremsstrahlung option for explaining the IR excess, since no silicate emission feature would then be expected. On the other hand, Hoard et al. (2009, 2010a, 2012) discuss possible scenarios in which the dust grain size distribution (if skewed toward large grains) could weaken the 10 $\mu \mathrm{m}$ silicate emission feature so that it is undetectable. As a rule of thumb, a spectral feature from dust at a particular wavelength is only present if the light-scattering grains are smaller than that wavelength (e.g., Spitzer 1978; Koike et al. 1980; D'Alessio et al. 2006; Voshchinnikov \& Henning 2008). Hence, while this property has been used to infer that dust grains in WD disks must have a typical size smaller than a few microns, it could also suggest that the dust grains in CVs are, on average, larger than $\sim 10 \mu \mathrm{m}$.

It is worth noting that the basic premise, that dust around CVs and single WDs should have similar observational properties, could be flawed, since these two types of object offer different environments and formation scenarios for dust. The origin of dust around WDs is established as a "top-down" process of collisional grinding of material from a tidally disrupted asteroid that was perturbed inside the Roche limit of the WD (Debes \& Sigurdsson 2002; Jura 2003). Yet, despite the fact that the total 
inferred dust mass in both WDs and CVs is similar (equivalent to a medium-large solar system asteroid, $\sim 10^{21}-10^{23} \mathrm{~g}$; this work, Hoard et al. 2009, Section 5.6.4 in Farihi 2011), tidal disruption of an asteroid by the WD cannot account for the formation of dust in circumbinary space around a CV. The scenario for dust formation in CVs would proceed through a "bottom-up" process of coagulation or clumping of smaller particles (gas and small dust grains; Lommen et al. 2010) that have escaped the inner binary. The specific formation path for very large $(\sim 10 \mu \mathrm{m})$ dust grains in circumbinary space around a CV remains an open question. See additional discussion of this topic in Hoard (2012).

A rather fundamental consideration that has been overlooked so far in this discussion is that we would also not expect to observe a strong (or any) $10 \mu \mathrm{m}$ silicate emission feature if the chemical composition of the dust is not predominantly silicate. For WDs, the dust has been shown to be chemically similar to the composition of the crust and mantle of terrestrial planets (Zuckerman et al. 2007, 2011; Farihi et al. 2011; Gänsicke et al. 2012) and, hence, is silicate-rich. This is consistent with its origin from a tidally disrupted asteroid. In $\mathrm{CVs}$, however, there is no such constraint on the origin of dust. For example, Albinson \& Evans (1987) suggest that under some circumstances the WDs and/or accretion disks in CVs might offer natural formation sites for carbon dust grains which could be subsequently expelled by radiation pressure. However, this process would seem to require the unlikely combination of a cool $(T \lesssim 12,000 \mathrm{~K})$ WD and a bright (hot) accretion disk (also see Johnson et al. 1993). Nonetheless, observations of the ejecta of Nova Herculis 1991 are consistent with the formation of carbon dust grains (Harrison \& Stringfellow 1994), while a mix of silicate and carbon/graphite grains are present in six novae observed by Smith et al. (1995). Counterbalancing this is the possibility that circumbinary dust production in CVs might be similar to that in the upper atmospheres and winds of asymptotic giant branch stars, which produce several times more silicate (mainly $\mathrm{SiC}$ ) grains than carbon (mainly amorphous graphite) grains (as deduced from compositional analyses of presolar grains in meteorites; Hoppe \& Zinner 2000; Draine 2003).

In the end, the cases that can currently be made in favor of (or in opposition to) either the bremsstrahlung or dust mechanisms as the origin of the observed IR excesses in CVs are rather ambiguous, largely circumstantial, and in need of stricter observational constraints.

\section{CONCLUSIONS}

The presence of an IR excess over the standard model accretion disk at wavelengths longer than $\approx 3-5 \mu \mathrm{m}$ is ubiquitous in our sample of 12 high mass transfer rate NLs. V592 Cas remains the NL with the most significant known IR excess out to wavelengths longer than $20 \mu \mathrm{m}$. Both V442 Oph and WX Ari have IR excesses brighter than even that of V592 Cas out to $7.9 \mu \mathrm{m}$ (IRAC-4), but we currently lack longer wavelength data to further constrain and characterize the source of the excess in these two systems. The SED of the excess component is similar in shape in all cases, and can be modeled with a circumbinary dust disk. We note that, in the context of modeling the IR SEDs of CVs, using a blackbody to represent the secondary star can lead to false-negatives for detection of IR excess, since this practice overestimates the brightness of a late-type star at long wavelengths. Despite the fact that it introduces complexity to the modeling process, it is advisable to use models that are as accurate and realistic as possible to represent all of the SED components.

In our sample, there is no significant correlation between the level of IR excess and the orbital period. Thus, while the presence of an IR excess is likely linked to the overall high mass transfer rate shared by all of our targets $\left(\gtrsim 10^{-9} M_{\odot} \mathrm{yr}^{-1}\right)$, its relative strength from system to system does not appear to be strongly dependent on the specific value of mass transfer rate over the ranges of orbital period (0.11-0.45 days; see Table 1) and mass transfer rate $\left(\sim 1-100 \times 10^{-9} M_{\odot} \mathrm{yr}^{-1}\right.$; see Tables 5 and 6) spanned by our sample of NLs. Coupled with the fact that the modeled total dust masses needed to reproduce the observed IR excesses are significantly smaller than the predicted threshold necessary to influence the secular evolution of CVs (also see discussion below), this implies that the presence of dust is not a dominant factor in driving that evolution. This conclusion is only valid if the mass transfer rate at a given orbital period is primarily determined by secular evolution (and, of course, if the mass transfer rates returned by the various accretion disk models are truly representative of the situation in these CVs).

On the other hand, the IR excess and system inclination show a statistically significant correlation. The increase in excess at $7.9 \mu \mathrm{m}$ with increasing inclination amounts to an $\approx 20 \%$ change from an inclination of $30^{\circ}$ (near face-on) to $90^{\circ}$ (edge-on). It is tempting to ascribe this relatively shallow trend to the changing view of the accretion disk at different inclinations (i.e., the hot, inner disk is increasingly self-occulted as inclination increases, leaving the accretion disk contribution to the SED to be dominated by the cooler outer disk and disk rim). However, comparison of model accretion disk SEDs computed at different inclinations suggests that this effect is likely not the sole factor in producing the observed level of change in IR excess.

There is an observational test that might be able to discriminate the location of the component producing the IR excess. The IR emission from circumbinary dust should not be affected by the primary eclipse in high inclination CVs, whereas IR emission originating from the inner binary (e.g., via bremsstrahlung or extremely cool material associated with the outer accretion disk) would likely be eclipsed to some extent, barring an extreme configuration in which the IR emission originates at large vertical distances above the accretion disk. While the latter scenario could be envisioned for free-free emission in an outflowing disk wind, the analysis presented here (see Section 4.1) implies that if the IR excess originates in a wind, then it most likely comes from the dense, accelerating base of the wind. Time-resolved mid-IR ( $\lambda \gtrsim 5 \mu \mathrm{m}$ ) observations of the eclipsing NLs in our sample (RW Tri, V347 Pup) or other high inclination NLs confirmed to have an IR excess would be helpful in this regard. Overall, however, the presence of a strong IR excess appears to go hand-in-hand with a high mass transfer rate, possibly via the formation of circumbinary dust in material that escapes the inner binary, as discussed in Hoard et al. (2009). This is not to say that CVs with lower mass transfer rates would be necessarily devoid of dust, but if present, then it is likely in a smaller amount, producing a weaker IR excess that is difficult to detect.

None of the CVs we observed show evidence in their SEDs for the presence of the $10 \mu \mathrm{m}$ emission feature that is the hallmark of the silicate dust found around numerous isolated WDs. There are a number of possible reasons why this feature could be absent even when dust is present in a CV (see Section 4.1.1), including predominantly large grain size and differences in chemical composition of the dust. The dust grain size has a strong effect on the total mass of dust required to produce an IR excess of 
a given amplitude. Thus, the lack of silicate emission at $10 \mu \mathrm{m}$ has potential implications for the amount of dust that could be present around these NLs (which, in turn, has implications for the potential effect of dust on the secular evolution of CVs). The total mass of silicate dust in our models that is required to reproduce the observed IR excesses in V592 Cas, IX Vel, UX UMa, and RW Sex is $\approx 1-2 \times 10^{21} \mathrm{~g}$ assuming a characteristic dust grain radius of $r_{\text {grain }}=1 \mu \mathrm{m}$ (see Table 5). For the case of large grains ( $r_{\text {grain }} \gtrsim 5 \mu \mathrm{m}$ ) corresponding to the absence of the $10 \mu \mathrm{m}$ silicate emission feature, the total dust masses would be larger by a factor of $\gtrsim 5$. At the low end $\left(r_{\text {grain }} \approx 5 \mu \mathrm{m}\right)$, the total mass is still insignificant compared to the prediction of $\gtrsim 10^{28} \mathrm{~g}$ required to influence CV secular evolution (Taam et al. 2003; Willems et al. 2007). Even at a reductio ad absurdum limit of centimeter-scale dust "pebbles" $\left(r_{\text {grain }} \approx 10^{4} \mu \mathrm{m}\right)$, the total dust mass is still 3 orders of magnitude too small to be important in this regard. While the absence of the $10 \mu \mathrm{m}$ silicate emission feature does not necessarily imply the absence of dust, it nonetheless remains true-and should be considered an observational priority - that detection of this feature in any quiescent system would be compelling evidence for the presence of dust in CVs.

This work is based on observations made with the Spitzer Space Telescope, which is operated by the Jet Propulsion Laboratory (JPL), California Institute of Technology (Caltech), under a contract with the National Aeronautics and Space Administration (NASA). Support for this work was provided by NASA. We acknowledge with thanks the variable star observations from the AAVSO International Database contributed by observers worldwide and used in this research. This work is also based on data, data products, and other resources obtained from: (1) The Two Micron All Sky Survey (2MASS), a joint project of the University of Massachusetts and the Infrared Processing and Analysis Center (IPAC)/Caltech, funded by NASA and the National Science Foundation (NSF). (2) NASA's Astrophysics Data System. (3) The NASA/IPAC Infrared Science Archive (IRSA), which is operated by JPL/Caltech, under a contract with NASA. (4) The SIMBAD database, operated at CDS, Strasbourg, France. (5) The Wide-field Infrared Survey Explorer (WISE), which is a joint project of the University of California, Los Angeles, and JPL/Caltech, funded by NASA.

Facilities: AAVSO, Spitzer, WISE

\section{APPENDIX}

\section{CONCISE HISTORIES OF TARGETS}

This appendix presents concise summaries of observational characteristics for the targets of our study, compiled from the literature. While not strictly necessary for interpreting our results, this information provides context for it.

\section{A.1. V592 Cas}

V592 Cas was discovered by Greenstein et al. (1970). Its blue color and spectral characteristics identified it as a NL. Its optical spectrum displays strong emission from He II $4686 \AA$ and the C III/N III/O II blend at $\approx 4640-4650 \AA{ }^{30}$ along with weak Balmer and He I emission lines (Downes et al. 1995). Huber et al.

\footnotetext{
30 We note in passing that although this broad emission feature found in many NL spectra is commonly referred to as the "Bowen blend," only the N III emission components actually result from the Bowen flourescence cascade (McClintock et al. 1975; Schachter et al. 1989).
}

(1998) performed the first time-resolved optical spectroscopic study of V592 Cas and used radial velocity measurements to determine an initial estimate of the orbital period, $P_{\text {orb }}=2.47 \mathrm{hr}$. This was later refined to $P_{\text {orb }}=2.76 \mathrm{hr}$ by Taylor et al. (1998) and Witherick et al. (2003). Although photometric observations show that it does not eclipse (inclination of $i=28^{\circ}$; Huber et al. 1998), V592 Cas does exhibit both positive and negative permanent superhumps (Taylor et al. 1998). These are believed to be caused by precession of an eccentric accretion disk and retrograde precession of a tilted accretion disk, respectively (e.g., Skillman et al. 1998; Kraicheva et al. 1999; Kim et al. 2009). Optical and UV spectroscopic observations demonstrated the presence of a wind, which manifests primarily as a $\mathrm{P}$ Cygni profile in the emission lines (Witherick et al. 2003; Prinja et al. 2004; Kafka et al. 2009). Kafka et al. (2009) showed that the wind is variable and reaches velocities up to $v_{\text {wind }} \sim 5000 \mathrm{~km} \mathrm{~s}^{-1}$ (which is greater than the WD escape velocity).

\section{A.2. IX Vel}

IX Vel was serendipitously discovered during a survey of OB stars (Garrison et al. 1977) and subsequently classified as a NL (Garrison et al. 1984). It displays low amplitude variability of $\sim 0.1-0.5$ mag on timescales ranging from minutes to years, and very broad, shallow Balmer absorption lines with emission cores. Photometric observations by Wargau et al. (1984) revealed flickering between $m_{\mathrm{pg}}=9.1$ and $10.0 \mathrm{mag}$, but their IR photometry showed no obvious eclipse, setting an upper limit to the inclination of roughly $65-70^{\circ}$. Linnell et al. (2007a) fit a BINSYN model to the far-UV (900-1700 A) SED of IX Vel (see Table 5). They found that the radial temperature profile of the accretion disk follows the standard model outside the innermost disk $\left(r_{\text {disk }}>4 R_{\mathrm{wd}}\right)$. The preliminary UV spectroscopic search for circumbinary disks by Belle et al. (2004) found no indication of absorption features related to circumbinary material around IX Vel.

\section{A.3. QU Car}

Although generally classified with the NLs, QU Car is also noted as a "V Sge" star. These objects have a massive WD and an evolved secondary star that allows very high mass transfer rates and leads to sustained nuclear burning on the surface of the WD (Steiner \& Diaz 1998). QU Car itself displays UV and optical nebular emission lines that likely originate in circumbinary material fed by the high velocity wind from the inner binary, and has been proposed as a prime candidate to become a Type Ia supernova (Kafka et al. 2008, 2012). Although its distance is somewhat uncertain, even at lower limit estimates QU Car is likely the intrinsically most luminous NL (Drew et al. 2003). Linnell et al. (2008b) fit a BINSYN model to the far-UV-optical (900-3000 ̊) SED of QU Car (see Table 5), and found that the hottest inner region of the disk is not wellrepresented by a standard model accretion disk. The preliminary UV spectroscopic search for circumbinary disks by Belle et al. (2004) found no indication of absorption features related to circumbinary material around QU Car.

\section{A.4. $R W$ Sex}

Greenstein \& Oke (1982) performed the first in-depth, multiwavelength investigation of RW Sex, noting weak X-ray emission, P Cygni profiles in the UV lines with terminal velocities on the order of WD escape velocity, and near-UV variability 
on timescales of several minutes. They suggest a substantial wind from the accretion disk is present (which could provide a source of raw material for the formation of dust). The blue optical spectrum of RW Sex shows broad, deep Balmer absorption lines with emission cores, and weak He II and $\mathrm{C}$ III/N III emission lines (Bolick et al. 1987). Optical radial velocity curves constructed by Beuermann et al. (1992a) identified these emission cores as originating on the heated inner face of the secondary star. Linnell et al. (2010) fit a BINSYN model to the farUV-optical (900-3000 ^) SED of RW Sex (see Table 5), and found that the hottest inner region of the disk is characterized by a temperature gradient shallower than the standard model (i.e., the inner disk remains hotter with increasing distance than the standard model). Greenstein \& Oke (1982) also found the SED of RW Sex to be flattened in the UV $(\lambda<5000 \AA)$.

\section{A.5. TT Ari}

TT Ari was found by Smak \& Stępień (1969) to show quasiperiodic and periodic variability on timescales of minutes to hours, as well as rapid flickering. Cowley et al. (1975) subsequently confirmed it as a CV through time-resolved spectroscopic observations. TT Ari is notable for displaying a wide range of variability on short timescales (e.g., Smak \& Stępień 1969; Mardirossian et al. 1980), bright and faint state transitions (i.e., VY Scl behavior-see Appendix A.9; e.g., Gänsicke et al. 1999; Kraicheva et al. 1999; Melikian et al. 2010), and both positive and negative superhumps (at different times). Emission line P Cygni profiles have been observed in its UV and optical spectra, likely indicative of an outflowing accretion disk wind (Melikian \& Karapetian 2004; Hutchings \& Cowley 2007).

\section{A.6. $R W$ Tri}

RW Tri is one of the earliest known CVs, discovered as an eclipsing binary more than 75 years ago (Protitch 1937, 1938). Groot et al. (2004) modeled RW Tri using optical (3600-7000 A) spectrophotometry, and found a radial temperature profile consistent with a standard steady state accretion disk (Frank et al. 2002). Rutten et al. (1992) reached the same conclusion via broad-band eclipse mapping. In a search for circumbinary material around several CVs, Dubus et al. (2004) found no need for an additional component to explain the near-IR SED of RW Tri.

\section{A.7. V347 Pup}

Buckley et al. (1990) used photometric and spectroscopic observations to identify V347 Pup as a deeply ( $\sim 3 \mathrm{mag})$, but only partially ( $V$-shaped profile), eclipsing NL. They noted that it is also a transient hard X-ray source, the counterpart to the HEAO 1 source 4U 0608-49. The accretion disk in V347 Pup appears to be at least partially self-occulting (e.g., see Mauche et al. 1994), suggesting that the contribution of the WD and hot inner disk to the system spectrum would likely be weaker than would otherwise be expected. Thoroughgood et al. (2005) made estimates of the stellar component masses and radii in V347 Pup, based on a time-resolved spectroscopic and photometric investigation. They reached a tentative conclusion that the secondary star is evolved.

\section{A.8. UX UMa}

UX UMa is an archetype of the NLs, and has been extensively studied since the mid-twentieth century (e.g., Walker 1953;
Johnson et al. 1954; Walker \& Herbig 1954). Warner \& Nather (1972) and Nather \& Robinson (1974) found low amplitude, $\approx 29$ s oscillations in the light curve of UX UMa, which they attributed to pulsations of the WD. Knigge et al. (1998) recovered these oscillations in Hubble Space Telescope time-series UV spectrophotometry, and discuss their origin in terms of a compact region of the inner accretion disk. Linnell et al. (2008a) fit a BINSYN model to the far-UV-optical (900-8000 A) SED of UX UMa (see Table 5). Although they found it challenging to produce a model consistent with all available observations, they note similarities of the accretion disk component with those in their models for IX Vel and QU Car; namely, that the radial temperature profile of the inner disk must be shallower (i.e., cooler) than the standard model (a feature also noted by Noebauer et al. 2010 in their Monte Carlo radiative transfer models of UX UMa). Nonetheless, Linnell et al. (2008a) conclude that a standard accretion disk model provides a reasonable fit to the observed SED of UX UMa. However, the model residuals from data sets obtained at different times suggest that the mass transfer rate in UX UMa is somewhat variable. Neustroev et al. (2011) also observed differences between optical spectra of UX UMa obtained in 1999 and 2008 that could be linked to a change in overall mass transfer rate at the two epochs.

\section{A.9. VY Scl}

VY Scl was first discovered in 1960 as an irregularly variable, faint blue star (Haro \& Chavira 1960). Its long-term photometric behavior (long stretches of stable brightness near 13th magnitude interspersed with drops to 18th magnitude lasting about 200 days) was noted as unique at the time (Warner \& van Citters 1974). While initially classified as an $\mathrm{R}$ Coronae Borealis star (Pişmiş 1972), Kraft \& Luyten (1964) suggested that VY Scl might be a $\mathrm{Z}$ Camelopardalis type $\mathrm{DN}^{31}$ that is mostly stuck in the "standstill" state, only dropping occasionally to its normal, faint quiescent state. Almost a decade later, Burrell \& Mould (1973) refuted the R CrB classification for VY Scl, and supported the Z Cam DN classification. However, they noted that the "chief difficulty" of this classification is that "VY Scl shows irregular minima, whereas (DNe) undergo outbursts." Hence, even with no comparable systems known at the time, Burrell \& Mould (1973) (as well as Kraft \& Luyten 1964 and Warner \& van Citters 1974) still identified in VY Scl the characteristic long-term photometric behavior that would later become the hallmark of the VY Scl stars. Members of this subtype of NL display intermittent, unpredictable drops to a faint state up to several magnitudes below their normal bright state level that last from weeks to months to years (Leach et al. 1999; Honeycutt \& Kafka 2004). This is believed to be caused by throttling of the accretion flow, likely due to solar-like cyclic magnetic activity on the secondary star (Livio \& Pringle 1994), or possibly a feedback loop in which irradiation of the secondary star by the hot inner accretion disk increases the mass transfer rate, which, in turn, inflates the outer disk and thereby reduces irradiation of the secondary star (Wu et al. 1995). Despite its status as a CV archetype, VY Scl itself is relatively little-studied, with only a few in-depth observational investigations in the past several decades (Hutchings \& Cowley 1984; Martínez-Pais et al. 2000; Hamilton \& Sion 2008).

\footnotetext{
31 Following normal outbursts, the Z Cam subclass of DN spends months to years in an intermediate brightness "standstill" state about 1 mag fainter than the outburst peak before dropping back to the quiescent state (Warner 2003).
} 


\section{A.10. V3885 Sgr}

V3885 Sgr was discovered as an emission line object (Bidelman et al. 1968); shortly thereafter, Cowley \& Hiltner (1969) published its complex optical spectrum, noting emission lines of $\mathrm{H}$ I, He I, and multiply ionized C, O, and Si. Ribeiro \& Diaz (2007) localized the main source of flickering in the $\mathrm{H} \alpha$ emission line to the irradiated face of the secondary star rather than the accretion disk. V3885 Sgr provided the first unambiguous detection of spiral waves in the accretion disk of a NL (Hartley et al. 2005); frequently found in DNe during outburst, the spiral waves originate from gravitational compression of the outer regions of an elliptical accretion disk by the WD as the disk rotates, which propagate inward as spiral shocks (e.g., see discussion in Hartley et al. 2005).

V3885 Sgr also has the distinction of being the first and, to date, only known NL with a reproducible radio detection (Körding et al. 2011); as such, it joins a small number of CVs with confirmed radio emission (primarily magnetic systems and outbursting classical and recurrent novae, but also including the DN SS Cyg during outburst; Körding et al. 2008). Radio emission possibly indicates the presence of jets producing optically thin synchrotron radiation. Linnell et al. (2009) fit a BINSYN model to the far-UV (900-1700 A) SED of V3885 Sgr (see Table 6), and found good agreement with a standard model accretion disk. The preliminary UV spectroscopic search for circumbinary disks by Belle et al. (2004) found no indication of absorption features related to circumbinary material around V3885 Sgr.

\section{A.11. V442 Oph}

V442 Oph was identified as a NL by Szkody \& Wade (1980) based on its photometric and spectroscopic properties. They noted that the He II $\lambda 4686$ emission is comparable in strength to H I. A follow-up investigation by Shafter \& Szkody (1983) utilized photometry and spectroscopy in the IR, optical, and UV. They measured an orbital period of $P_{\text {orb }}=0.1403 \mathrm{~d}(=7.13$ cycles $\mathrm{d}^{-1}$ ) from $\mathrm{H}$ i and He II $\lambda 4686$ radial velocity curves and found a roughly sinusoidal variation in the $J$-band light curve consistent with this period (presumably caused by the changing visibility of the irradiation-heated inner face of the secondary star). However, extensive time series optical photometry did not reveal any evidence of an eclipse, implying a system inclination of $i \lesssim 65^{\circ}$. Hoard et al. (2000) suggested that V442 Oph shared many of the observational characteristics of the SW Sex stars. They used extensive $\mathrm{H} \alpha$ radial velocity measurements to demonstrate that the true orbital period of V442 Oph is $0.12435(7)$ days $\left(=8.06\right.$ cycles days $\left.{ }^{-1}\right)$, with the longer Shafter \& Szkody (1983) period being a 1 day alias. Diaz (2001) subsequently refined the orbital period to 0.1243301(9) days using additional $\mathrm{H} \alpha$ radial velocity measurements. Patterson et al. (2002) identified low amplitude, persistent superhumps (both positive and negative) in V442 Oph, and suggested that persistent superhumps might be present in all SW Sex stars.

\section{A.12. WX Ari}

There are few dedicated observational investigations of WX Ari. Beuermann et al. (1992b) obtained time-resolved optical spectra and determined its orbital period from radial velocity measurements. They suggested that WX Ari was a member of the then recently proposed SW Sex class, but noted that it did not appear to eclipse (all known SW Sex stars at the time were deeply eclipsing). In a short note, Hellier et al.
(1994) concluded from several hours of $V$-band photometry that WX Ari did not eclipse. However, Rodríguez-Gil et al. (2000) obtained a longer set of $R$-band photometry and found that WX Ari is at an intermediate inclination $\left(i \sim 72^{\circ}\right)$ with shallow $(\Delta R \sim 0.15 \mathrm{mag})$ partial eclipses. Ballouz \& Sion (2009) favor a lower inclination $\left(i \sim 60^{\circ}\right)$ based on model fits to the UV spectrum of WX Ari.

\section{REFERENCES}

Albinson, J. S., \& Evans, A. 1987, Ap\&SS, 131, 443

Allard, F., Allard, N. F., Homeier, D., et al. 2007, A\&A, 474, L21

Allard, F., Guillot, T., Ludwig, H.-G., et al. 2003, in IAU Symp. 211, Brown Dwarfs, ed. E. Martín (San Francisco, CA: ASP), 325

Allard, F., Homeier, D., \& Freytag, B. 2012, RSPTA, 370, 2765

Ballouz, R.-L., \& Sion, E. M. 2009, ApJ, 697, 1717

Barlow, M. J., \& Cohen, M. 1977, ApJ, 213, 737

Belle, K. E., Sanghi, N., Howell, S. B., Holberg, J. B., \& Williams, P. T. 2004, AJ, 128,448

Beuermann, K., Buhlmann, J., Diese, J., et al. 2011, A\&A, 526, A53

Beuermann, K., Stasiewski, U., \& Schwope, A. D. 1992a, A\&A, 256, 433

Beuermann, K., \& Thomas, H.-C. 1990, A\&A, 230, 326

Beuermann, K., Thorstensen, J. R., Schwope, A. D., Ringwald, F. A., \& Sahin, H. 1992b, A\&A, 256, 442

Bidelman, W. P., MacConnell, D. J., \& Bond, H. E. 1968, IAUC, 2089, 0

Bisikalo, D. V. 2009, in AIP Conf. Ser. 1121, Space Plasma Physics: School of Space Plasma Physics, ed. I. Zhelyazkov (Melville, NY: AIP), 3

Bisikalo, D. V. 2011, in EAS Publications Series 44, Proc. of JENAM 2008: Grand Challenges in Computational Astrophysics, ed. H. Wozniak \& G. Hensler (Cambridge: Cambridge Univ. Press), 121

Bode, M. F. 1982, VA, 26, 369

Bolick, U., Beuermann, K., Bruch, A., \& Lenzen, R. 1987, Ap\&SS, 130, 175

Bours, M. C. P., Toonen, S., \& Nelemans, G. 2013, A\&A, 552, A24

Brinkworth, C. S., Marsh, T. R., Dhillon, V. S., \& Knigge, C. 2006, MNRAS, 365,287

Buckley, D. A. H., Sullivan, D. J., Remillard, R. A., Tuohy, I. R., \& Clark, M. 1990, ApJ, 355, 617

Burrell, J. F., \& Mould, J. R. 1973, PASP, 85, 627

Cannizzo, J. K., Shafter, A. W., \& Wheeler, J. C. 1988, ApJ, 333, 227

Carciofi, A. C., Bjorkman, J. E., \& Magalhães, A. M. 2004, ApJ, 604, 238

Carey, S., Ingalls, J., Hora, J., et al. 2012, Proc. SPIE, 8442, $1 Z$

Ciardi, D. R., Wachter, S., Hoard, D. W., Howell, S. B., \& van Belle, G. T. 2006, AJ, 132, 1989

Cohen, M., Wheaton, W. A., \& Megeath, S. T. 2003, AJ, 126, 1090

Cowley, A. P., Crampton, D., Hutchings, J. B., \& Marlborough, J. M. 1975, ApJ, 195,413

Cowley, A. P., \& Hiltner, W. A. 1969, A\&A, 3, 372

D’Alessio, P., Calvet, N., Hartmann, L., Franco-Hernández, R., \& Servín, H. 2006, ApJ, 638, 314

Debes, J. H., \& Sigurdsson, S. 2002, ApJ, 572, 556

Dhillon, V. S., Littlefair, S. P., Howell, S. B., et al. 2000, MNRAS, 314, 826

Dhillon, V. S., Smith, D. A., \& Marsh, T. R. 2013, MNRAS, 428, 3559

Diaz, M. P. 2001, ApJL, 553, L177

Diaz, M. P., \& Hubeny, I. 1999, ApJ, 523, 786

Downes, R., Hoard, D. W., Szkody, P., \& Wachter, S. 1995, AJ, 110, 1824

Downes, R. A., Webbink, R. F., Shara, M. M., et al. 2001, PASP, 113, 764

Doyle, L. R., Carter, J. A., Fabrycky, D. C., et al. 2011, Sci, 333, 1602

Draine, B. T. 1981, ApJ, 245, 880

Draine, B. T. 2003, ARA\&A, 41, 241

Drew, J. E., Hartley, L. E., Long, K. S., \& van der Walt, J. 2003, MNRAS, 338,401

Dubus, G., Campbell, R., Kern, B., Taam, R. E., \& Spruit, H. C. 2004, MNRAS, 349,869

Dubus, G., Taam, R. E., \& Spruit, H. C. 2002, ApJ, 569, 395

Dwek, E., Sellgren, K., Soifer, B. T., \& Werner, M. W. 1980, ApJ, 238, 140

Evans, A. 1997, Ap\&SS, 251, 293

Evans, A. 2001, Ap\&SS, 275, 131

Evans, A., \& Gehrz, R. D. 2012, BASI, 40, 213

Evans, A., Woodward, C. E., Helton, L. A., et al. 2007, ApJL, 671, L157

Farihi, J. 2011, in White Dwarf Atmospheres and Circumstellar Environments, ed. D. W. Hoard (Berlin: Wiley), 117

Farihi, J., Brinkworth, C. S., Gänsicke, B. T., et al. 2011, ApJL, 728, L8

Frank, J., King, A., \& Raine, D. J. 2002, Accretion Power in Astrophysics (Cambridge: Cambridge Univ. Press)

Gänsicke, B. T., Koester, D., Farihi, J., et al. 2012, MNRAS, 424, 333 
Gänsicke, B. T., Sion, E. M., Beuermann, K., et al. 1999, A\&A, 347, 178 Garrison, R. F., Hiltner, W. A., \& Schild, R. E. 1977, ApJS, 35, 111 Garrison, R. F., Schild, R. E., Hiltner, W. A., \& Krzeminski, W. 1984, ApJL, 276, L13

Gautier, T. N., III, Rieke, G. H., Stansberry, J., et al. 2007, ApJ, 667, 527

Gilliland, R. L., \& Phillips, M. M. 1982, ApJ, 261, 617

Goździewski, K., Nasiroglu, I., Słowikowska, A., et al. 2012, MNRAS, 425,930

Greenstein, J. L., \& Oke, J. B. 1982, ApJ, 258, 209

Greenstein, J. L., Sargent, A. I., \& Haug, U. 1970, A\&A, 7, 1

Greiner, J., Schwarz, R., Tappert, C., et al. 2010, AN, 331, 227

Groot, P. J., Rutten, R. G. M., \& van Paradijs, J. 2004, A\&A, 417, 283

Hameury, J.-M., Menou, K., Dubus, G., Lasota, J.-P., \& Hure, J.-M. 1998, MNRAS, 298, 1048

Hamilton, R. T., \& Sion, E. M. 2008, PASP, 120, 165

Haro, G., \& Chavira, E. 1960, BOTT, 2, 11

Harrison, T. E., Hamilton, R. T., Tappert, C., Hoffman, D. I., \& Campbell, R. K. 2013, AJ, 145, 19

Harrison, T. E., Osborne, H. L., \& Howell, S. B. 2004, AJ, 127, 3493

Harrison, T. E., Osborne, H. L., \& Howell, S. B. 2005, AJ, 129, 2400

Harrison, T. E., \& Stringfellow, G. S. 1994, ApJ, 437, 827

Hartley, L. E., Murray, J. R., Drew, J. E., \& Long, K. S. 2005, MNRAS, 363,285

Hellier, C., Ringwald, F. A., \& Robinson, E. L. 1994, A\&A, 289, 148

Hillwig, T., Livio, M., \& Honeycutt, R. K. 2004, PASP, 116, 397

Hinse, T. C., Lee, J. W., Goździewski, K., et al. 2012, MNRAS, 420, 3609

Hoard, D. W. 2012, MmSAI, 83, 490

Hoard, D. W., Howell, S. B., Brinkworth, C. S., Ciardi, D. R., \& Wachter, S. 2007, ApJ, 671, 734

Hoard, D. W., Howell, S. B., \& Stencel, R. E. 2010a, ApJ, 714, 549

Hoard, D. W., Kafka, S., Wachter, S., et al. 2009, ApJ, 693, 236

Hoard, D. W., Ladjal, D., Stencel, R. E., \& Howell, S. B. 2012, ApJL, 748, L28

Hoard, D. W., Lu, T.-N., Knigge, C., et al. 2010b, AJ, 140, 1313

Hoard, D. W., Szkody, P., Froning, C. S., Long, K. S., \& Knigge, C. 2003, AJ, 126,2473

Hoard, D. W., Thorstensen, J. R., \& Szkody, P. 2000, ApJ, 537, 936

Honeycutt, R. K., \& Kafka, S. 2004, AJ, 128, 1279

Honeycutt, R. K., Schlegel, E. M., \& Kaitchuck, R. H. 1986, ApJ, 302, 388

Hoppe, P., \& Zinner, E. 2000, JGR, 105, 10371

Horner, J., Marshall, J. P., Wittenmyer, R. A., \& Tinney, C. G. 2011, MNRAS, 416, L11

Howell, S. B., Nelson, L. A., \& Rappaport, S. 2001, ApJ, 550, 897

Howell, S. B., Szkody, P., Sonneborn, G., et al. 1995, ApJ, 453, 454

Huber, M. E., Howell, S. B., Ciardi, D. R., \& Fried, R. 1998, PASP, 110, 784

Hutchings, J. B., \& Cowley, A. P. 1984, PASP, 96, 559

Hutchings, J. B., \& Cowley, A. P. 2007, AJ, 133, 1204

Jarrett, T. H., Cohen, M., Masci, F., et al. 2011, ApJ, 735, 112

Johnson, D. J., Friedlander, M. W., \& Katz, J. I. 1993, ApJ, 407, 714

Johnson, H. L., Perkins, B., \& Hiltner, W. A. 1954, ApJS, 1, 91

Jura, M. 2003, ApJL, 584, L91

Jura, M., Farihi, J., \& Zuckerman, B. 2009, AJ, 137, 3191

Jura, M., Farihi, J., Zuckerman, B., \& Becklin, E. E. 2007, AJ, 133, 1927

Kafka, S., Anderson, R., \& Honeycutt, R. K. 2008, AJ, 135, 1649

Kafka, S., Hoard, D. W., Honeycutt, R. K., \& Deliyannis, C. P. 2009, AJ, 137,197

Kafka, S., Honeycutt, R. K., \& Williams, R. 2012, MNRAS, 425, 1585

Kim, Y., Andronov, I. L., Cha, S. M., Chinarova, L. L., \& Yoon, J. N. 2009, A\&A, 496, 765

Knigge, C. 2006, MNRAS, 373, 484

Knigge, C., Baraffe, I., \& Patterson, J. 2011, ApJS, 194, 28

Knigge, C., Drake, N., Long, K. S., et al. 1998, ApJ, 499, 429

Kobayashi, H., Kimura, H., Watanabe, S.-i., Yamamoto, T., \& Müller, S. 2011, EP\&S, 63, 1067

Koike, C., Hasegawa, H., \& Manabe, A. 1980, Ap\&SS, 67, 495

Körding, E. G., Knigge, C., Tzioumis, T., \& Fender, R. 2011, MNRAS, 418, L129

Körding, E., Rupen, M., Knigge, C., et al. 2008, Sci, 320, 1318

Kraft, R. P., \& Luyten, W. J. 1964, in Proc. of the First Conference on Faint Blue Stars, ed. W. J. Luyten (Minneapolis: Univ. Minnesota), 77

Kraicheva, Z., Stanishev, V., Genkov, V., \& Iliev, L. 1999, A\&A, 351, 607

Lasota, J.-P. 2001, NewAR, 45, 449

Leach, R., Hessman, F. V., King, A. R., Stehle, R., \& Mattei, J. 1999, MNRAS, 305,225

Lee, J. W., Kim, S.-L., Kim, C.-H., et al. 2009, AJ, 137, 3181

Linnell, A. P., DeStefano, P., \& Hubeny, I. 2012, PASP, 124, 885

Linnell, A. P., Godon, P., Hubeny, I., Sion, E. M., \& Szkody, P. 2007a, ApJ, 662,1204
Linnell, A. P., Godon, P., Hubeny, I., Sion, E. M., \& Szkody, P. 2008a, ApJ 688,568

Linnell, A. P., Godon, P., Hubeny, I., Sion, E. M., \& Szkody, P. 2010, ApJ, 719,271

Linnell, A. P., Godon, P., Hubeny, I., et al. 2008b, ApJ, 676, 1226

Linnell, A. P., Godon, P., Hubeny, I., et al. 2009, ApJ, 703, 1839

Linnell, A. P., Hoard, D. W., Szkody, P., et al. 2007b, ApJ, 654, 1036

Linnell, A. P., \& Hubeny, I. 1996, ApJ, 471, 958

Livio, M., \& Pringle, J. E. 1994, ApJ, 427, 956

Lommen, D. J. P., van Dishoeck, E. F., Wright, C. M., et al. 2010, A\&A, 515, A77

Mardirossian, F., Mezzetti, M., Pucillo, M., et al. 1980, A\&A, 85, 29

Marsh, T. R., Parsons, S. G., Bours, M. C. P., et al. 2014, MNRAS, 437, 475

Martínez-Pais, I. G., Martín-Hernández, N. L., Casares, J., \& Rodríguez-Gil, P. 2000, ApJ, 538, 315

Mauche, C. W., Raymond, J. C., Buckley, D. A. H., et al. 1994, ApJ, 424, 347

McArthur, B. E., Benedict, G. F., Lee, J., et al. 1999, ApJL, 520, L59

McClintock, J. E., Canizares, C. R., \& Tarter, C. B. 1975, ApJ, 198, 641

Melikian, N. D., \& Karapetian, A. A. 2004, Ap, 47, 462

Melikian, N. D., Tamazian, V. S., Docobo, J. A., et al. 2010, Ap, 53, 373

Nather, R. E., \& Robinson, E. L. 1974, ApJ, 190, 637

Neustroev, V. V., Suleimanov, V. F., Borisov, N. V., Belyakov, K. V., \& Shearer, A. 2011, MNRAS, 410, 963

Noebauer, U. M., Long, K. S., Sim, S. A., \& Knigge, C. 2010, ApJ, 719, 1932

Orosz, J. A., \& Wade, R. A. 2003, ApJ, 593, 1032

Orosz, J. A., Welsh, W. F., Carter, J. A., et al. 2012a, ApJ, 758, 87

Orosz, J. A., Welsh, W. F., Carter, J. A., et al. 2012b, Sci, 337, 1511

Osaki, Y. 1996, PASP, 108, 39

Parsons, S. G., Marsh, T. R., Bours, M. C. P., et al. 2014, MNRAS, 438, L91

Parsons, S. G., Marsh, T. R., Copperwheat, C. M., et al. 2010, MNRAS, 407, 2362

Patten, B. M., Stauffer, J. R., Burrows, A., et al. 2006, ApJ, 651, 502

Patterson, J. 1984, ApJS, 54, 443

Patterson, J., Fenton, W. H., Thorstensen, J. R., et al. 2002, PASP, 114, 1364

Pişmiş, P. 1972, BOTT, 6, 197

Pollack, J. B., Hollenbach, D., Beckwith, S., et al. 1994, ApJ, 421, 615

Poole, T., Mason, K. O., Ramsay, G., Drew, J. E., \& Smith, R. C. 2003, MNRAS, 340,499

Potter, S. B., Romero-Colmenero, E., Ramsay, G., et al. 2011, MNRAS, 416, 2202

Prinja, R. K., Knigge, C., Witherick, D. K., Long, K. S., \& Brammer, G. 2004, MNRAS, 355, 137

Prinja, R. K., \& Rosen, R. 1995, MNRAS, 273, 461

Proga, D., Kallman, T. R., Drew, J. E., \& Hartley, L. E. 2002, ApJ, 572, 382

Protitch, M. 1937, BABel, 9, 38

Protitch, M. 1938, AN, 266, 95

Puebla, R. E., Diaz, M. P., Hillier, D. J., \& Hubeny, I. 2011, ApJ, 736, 17

Puebla, R. E., Diaz, M. P., \& Hubeny, I. 2007, AJ, 134, 1923

Qian, S.-B., Liao, W.-P., Zhu, L.-Y., \& Dai, Z.-B. 2010a, ApJL, 708, L66

Qian, S.-B., Liao, W.-P., Zhu, L.-Y., et al. 2010b, MNRAS, 401, L34

Qian, S.-B., Liu, L., Liao, W.-P., et al. 2011, MNRAS, 414, L16

Qian, S.-B., Liu, L., Zhu, L.-Y., et al. 2012a, MNRAS, 422, L24

Qian, S.-B., Zhu, L.-Y., Dai, Z.-B., et al. 2012b, ApJL, 745, L23

Reach, W. T., Lisse, C., von Hippel, T., \& Mullally, F. 2009, ApJ, 693, 697

Ribeiro, F. M. A., \& Diaz, M. P. 2007, AJ, 133, 2659

Rodríguez-Gil, P., Casares, J., Dhillon, V. S., \& Martínez-Pais, I. G. 2000, A\&A, 355,181

Rutten, R. G. M., van Paradijs, J., \& Tinbergen, J. 1992, A\&A, 260, 213

Schachter, J., Filippenko, A. V., \& Kahn, S. M. 1989, ApJ, 340, 1049

Schwarz, R., Schwope, A. D., Vogel, J., et al. 2009, A\&A, 496, 833

Shafter, A. W., \& Szkody, P. 1983, PASP, 95, 509

Skillman, D. R., Harvey, D. A., Patterson, J., et al. 1998, ApJL, 503, L67

Skrutskie, M. F., Cutri, R. M., Stiening, R., et al. 2006, AJ, 131, 1163

Smak, J., \& Stępień, K. 1969, in Communications of the Konkoly Observatory 65, Non-periodic Phenomena in Variable Stars, ed. L. Petre (Budapest: Academic), 355

Smith, C. H., Aitken, D. K., Roche, P. F., \& Wright, C. M. 1995, MNRAS, 277, 259

Spitzer, L. 1978, Physical Processes in the Interstellar Medium (New York: Wiley)

Spruit, H. C., \& Taam, R. E. 2001, ApJ, 548, 900

Steiner, J. E., \& Diaz, M. P. 1998, PASP, 110, 276

Stetson, P. B. 1987, PASP, 99, 191

Sytov, A. Y., Bisikalo, D. V., Kaigorodov, P. V., \& Boyarchuk, A. A. 2009, ARep, 53, 223

Sytov, A. Y., Kaigorodov, P. V., Bisikalo, D. V., Kuznetsov, O. A., \& Boyarchuk, A. A. 2007, ARep, 51, 836 
Szkody, P. 1977, ApJ, 217, 140

Szkody, P., \& Piche, F. 1990, ApJ, 361, 235

Szkody, P., \& Wade, R. A. 1980, PASP, 92, 806

Taam, R. E., Sandquist, E. L., \& Dubus, G. 2003, ApJ, 592, 1124

Taylor, C. J., Thorstensen, J. R., Patterson, J., et al. 1998, PASP, 110, 1148

Taam, R. E., \& Spruit, H. C. 2001, ApJ, 561, 329

Thoroughgood, T. D., Dhillon, V. S., Steeghs, D., et al. 2005, MNRAS, 357,881

Thorstensen, J. R., Ringwald, F. A., Wade, R. A., Schmidt, G. D., \& Norsworthy, J. E. 1991, AJ, 102, 272

van Hamme, W. 1993, AJ, 106, 2096

Voshchinnikov, N. V., \& Henning, T. 2008, A\&A, 483, L9

Wade, R. A. 1988, ApJ, 335, 394

Walker, M. F. 1953, PASP, 65, 206

Walker, M. F., \& Herbig, G. H. 1954, ApJ, 120, 278

Wargau, W., Drechsel, H., Rahe, J., Bruch, A., \& Schoembs, R. 1984, Ap\&SS, 99,145

Warner, B. 2003, Cataclysmic Variable Stars (Cambridge: Cambridge Univ. Press)
Warner, B., \& Nather, R. E. 1972, MNRAS, 159, 429

Warner, B., \& van Citters, G. W. 1974, Obs, 94, 116

Welsh, W. F., Orosz, J. A., Carter, J. A., et al. 2012, Natur, 481, 475

Willems, B., Kolb, U., Sandquist, E. L., Taam, R. E., \& Dubus, G. 2005, ApJ, 635,1263

Willems, B., Taam, R. E., Kolb, U., Dubus, G., \& Sandquist, E. L. 2007, ApJ, 657,465

Witherick, D. K., Prinja, R. K., Howell, S. B., \& Wagner, R. M. 2003, MNRAS, 346,861

Wittenmyer, R. A., Horner, J., Marshall, J. P., Butters, O. W., \& Tinney, C. G. 2012, MNRAS, 419, 3258

Wright, A. E., \& Barlow, M. J. 1975, MNRAS, 170, 41

Wright, E. L., Eisenhardt, P. R. M., Mainzer, A. K., et al. 2010, AJ, 140,1868

Wu, X., Li, Z., Ding, Y., Zhang, Z., \& Li, Z. 2002, ApJ, 569, 418

Wu, K., Wickramasinghe, D. T., \& Warner, B. 1995, PASA, 12, 60

Zuckerman, B., Koester, D., Dufour, P., et al. 2011, ApJ, 739, 101

Zuckerman, B., Koester, D., Melis, C., Hansen, B. M., \& Jura, M. 2007, ApJ, 671,872 